\title{
Aza-Nazarov Reaction and the Role of Superelectrophiles.
}

Douglas A. Klumpp ${ }^{\dagger *}$, Yiliang Zhang ${ }^{\dagger}$, Matthew J. O’Connor ${ }^{\dagger}$, Pierre M. Esteves ${ }^{\ddagger}$, and Leonardo S. de Almeida

${ }^{\dagger}$ Department of Chemistry, Northern Illinois University, DeKalb, Illinois, 60115, and "Instituto de Quimica, Universidade Federal do Rio de Janeiro, Cidade Universitaria, CT Bloco A, 21949-900, Rio de Janeiro-RJ, Brazil.

S-1 Index

S-2 to S-5 Experimental procedures and characterization data

S-6 to S-19 $\quad{ }^{1} \mathrm{H}$ and ${ }^{13} \mathrm{C}$ NMR spectra

S-20 References to known compounds

S-21 to S-30 Computational methods, results, tables, and figures. 
All reactions were performed with oven-dried glassware, and the moisture-sensitive reactions were setup under Ar. Trifluoromethanesulfonic acid was distilled under an Ar atmosphere prior to use. All commercially available compounds and anhydrous solvents were used as received. Imines were purchased from commercial suppliers, although salt 21 was prepared from 3,4-dihydroisoquinoline. ${ }^{1}{ }^{1} \mathrm{H}$ and ${ }^{13} \mathrm{C}$ NMR spectra were recorded on Brüker 200 or $500 \mathrm{MHz}$ spectrometer and low resolution mass spectra were obtained from a capillary GC (DB-5 column) equipped with a mass selective detector. Highresolution mass spectra were obtained from the Analytical Services Department at University of Illinois. Photolysis reactions were done using a $450 \mathrm{~W}$ medium pressure $\mathrm{Hg}$ vapor lamp (pyrex filter) and a photochemical reactor assembly from Ace Glass.

General procedure for the preparation of cyclization products: A $25 \mathrm{~mL}$ roundbottom flask containing $1.0 \mathrm{mmol}$ imine in $5 \mathrm{~mL} \mathrm{CH}_{2} \mathrm{Cl}_{2}$ is added 1.0 equivalent carboxylic acid chloride slowly. After stirring at room temperature for $2 \mathrm{hrs}, 5$ or more equivalents freshly distilled triflic acid is added (slowly). Evolution of $\mathrm{HCl}$ gas requires that the reaction flask have an outlet or vent. The reaction mixture is stirred at room temperature for at least $2 \mathrm{hrs}$. Then the reaction mixture is poured onto ice and extracted with $\mathrm{CHCl}_{3}$. Following by washing with brine, the organic layer is dried over anhydrous $\mathrm{Na}_{2} \mathrm{SO}_{4}$. If necessary, the products are purified by flash column chromatography (hexanes:ether).

Alternatively, acylation of the imine can be done by reaction of $1.0 \mathrm{mmol}$ of the imine with $1.0 \mathrm{mmol}$ of the carboxylic acid chloride in $5 \mathrm{~mL}$ of anhydrous $\mathrm{CH}_{3} \mathrm{CN}$. After stirring $2 \mathrm{hrs}$ at room temperature, the solvent is removed by vacuum. As described above, the triflic acid is then added and the reaction is later quenched. Product isolation is done as above.

5-Methoxy-2-methyl-3-phenyl-2,3-dihydro-isoindol-1-one (7b): White solid, mp: 110$114^{\circ} \mathrm{C} . \quad{ }^{1} \mathrm{H}$ NMR $\left(\mathrm{CDCl}_{3}, 200 \mathrm{MHz}\right) \delta 2.92(\mathrm{~s}, 3 \mathrm{H}), 3.73(\mathrm{~s}, 3 \mathrm{H}), 5.25(\mathrm{~s}, 1 \mathrm{H}), 6.61-6.64$ $(\mathrm{m}, 1 \mathrm{H}), 6.87-6.97(\mathrm{~m}, 1 \mathrm{H}), 7.10-7.16(\mathrm{~m}, 2 \mathrm{H}), 7.30-7.36(\mathrm{~m}, 3 \mathrm{H}), 7.79(\mathrm{~d}, 1 \mathrm{H}, J=8.4$ $\mathrm{Hz}) .{ }^{13} \mathrm{C} \mathrm{NMR}\left(\mathrm{CDCl}_{3}, 50 \mathrm{MHz}\right) \delta 27.4,55.5,66.3,107.8,114.8,124.1,124.7,127.4$, 128.6, 129.1, 137.0, 148.3, 162.9, 168.7. Low resolution mass spectra (EI): m/z: 253 $(\mathrm{M}+), 222,176,152,118,77$. HRMS for $\mathrm{C}_{16} \mathrm{H}_{15} \mathrm{O}_{2} \mathrm{~N}$, calcd: 253.1103, found: 253.1109 .

5-Chloro-2-methyl-3-phenyl-2,3-dihydro-isoindol-1-one (7c): White solid, mp: 169$171^{\circ} \mathrm{C} .{ }^{1} \mathrm{H}$ NMR $\left(\mathrm{CDCl}_{3}, 200 \mathrm{MHz}\right) \delta 2.97(\mathrm{~s}, 3 \mathrm{H}), 5.33(\mathrm{~s}, 1 \mathrm{H}), 7.14-7.17(\mathrm{~m}, 3 \mathrm{H})$, 7.36-7.46 (m, 4H), $7.81(\mathrm{~d}, 1 \mathrm{H}, J=8.1 \mathrm{~Hz}) .{ }^{13} \mathrm{C} \mathrm{NMR}\left(\mathrm{CDCl}_{3}, 50 \mathrm{MHz}\right) \delta 27.6,66.2$, 123.4, 124.7, 127.4, 129.0, 129.3, 130.2, 136.2, 138.0, 147.6, 167.6. Low resolution mass spectrum (EI), m/z: 257(M+), 222, 180, 118, 77. 
4,6-Dimethoxy-2-methyl-3-phenyl-2,3-dihydro-isoindol-1-one (9a): White solid, mp: 123-125 ${ }^{\circ} \mathrm{C} .{ }^{1} \mathrm{H}$ NMR $\left(\mathrm{CDCl}_{3}, 500 \mathrm{MHz}\right) \delta 2.88$ (s, 3H), 3.59 (s, 3H), $3.84(\mathrm{~s}, 3 \mathrm{H}), 5.26(\mathrm{~s}$, $1 \mathrm{H}), 6.48(\mathrm{~d}, J=1.1 \mathrm{~Hz}, 1 \mathrm{H}), 6.99(\mathrm{~d}, J=1.1 \mathrm{~Hz}, 1 \mathrm{H}), 7.10-7.12(\mathrm{~m}, 2 \mathrm{H}), 7.24-7.33(\mathrm{~m}$, $3 \mathrm{H}) .{ }^{13} \mathrm{C} \mathrm{NMR}\left(\mathrm{CDCl}_{3}, 125 \mathrm{MHz}\right) \delta 27.4,55.5,55.8,64.6,97.8,102.7,126.6,127.7$, 128.1, 128.5, 134.2, 136.5, 155.3, 162.0. Low resolution mass spectra (EI): m/z: 283 $(\mathrm{M}+), 252,206,118,77$. HRMS for $\mathrm{C}_{17} \mathrm{H}_{17} \mathrm{O}_{3} \mathrm{~N}$, calcd: 283.1208, found: 283.1213 .

4,6-Dichloro-2-methyl-3-phenyl-2,3-dihydro-isoindol-1-one (9b): White solid, mp: 174-175 ${ }^{\circ}$; ${ }^{1} \mathrm{H}$ NMR $\left(\mathrm{CDCl}_{3}, 500 \mathrm{MHz}\right) \delta 3.00(\mathrm{~s}, 3 \mathrm{H}), 5.34(\mathrm{~s}, 1 \mathrm{H}), 7.16-7.19(\mathrm{~m}, 2 \mathrm{H})$, 7.29-7.49 (m, 4H), $7.84(\mathrm{~d}, J=8.1 \mathrm{~Hz}, 1 \mathrm{H}), 8.07(\mathrm{~d}, J=8.3 \mathrm{~Hz}, 1 \mathrm{H}) \cdot{ }^{13} \mathrm{C} \mathrm{NMR}\left(\mathrm{CDCl}_{3}\right.$, $125 \mathrm{MHz}$ ) $\delta$ 27.6, 66.2, 123.4, 124.7, 127.4 (two carbons), 129.0, 129.3, 130.2, 136.2, 138.0, 147.6, 167.7. Low resolution mass spectra (EI): m/z: 291 and $293(\mathrm{M}+), 256,214$, $118,77$.

4,5,6-Trimethoxy-2-methyl-3-phenyl-2,3-dihydro-isoindol-1-one (11): White solid, mp: $100-104^{\circ} \mathrm{C} .{ }^{1} \mathrm{H}$ NMR $\left(\mathrm{CDCl}_{3}, 500 \mathrm{MHz}\right) \delta 2.89$ (s, 3H), $3.28(\mathrm{~s}, 3 \mathrm{H}), 3.83(\mathrm{~s}, 3 \mathrm{H})$, $3.91(\mathrm{~s}, 3 \mathrm{H}), 5.29(\mathrm{~s}, 1 \mathrm{H}), 7.14(\mathrm{~d}, 2 \mathrm{H}, J=7.6 \mathrm{~Hz}), 7.22(\mathrm{~s}, 1 \mathrm{H}), 7.27-7.33(\mathrm{~m}, 3 \mathrm{H}) .{ }^{13} \mathrm{C}$ NMR $\left(\mathrm{CDCl}_{3}, 125 \mathrm{MHz}\right) \delta 27.4,56.4,60.1,60.8,64.6,101.8,127.1,127.7,128.4,128.8$, 131.7, 136.9, 145.4, 148.3, 155.1, 168.3. Low resolution mass spectra (EI): m/z: 313 $(\mathrm{M}+), 282,236,118,77$. HRMS for $\mathrm{C}_{18} \mathrm{H}_{19} \mathrm{O}_{4} \mathrm{~N}$, calcd: 313.1314 , found: 313.1316 .

2,3-Bis-(4-fluoro-phenyl)-4,5,6-trimethoxy-2,3-dihydro-isoindol-1-one (13): White solid, mp: $130-132^{\circ} \mathrm{C} .{ }^{1} \mathrm{H}$ NMR $\left(\mathrm{CDCl}_{3}, 500 \mathrm{MHz}\right) \delta 3.38$ (s, 3H), 3.88 (s, 3H), 3.94 (s, $3 \mathrm{H}), 6.00(\mathrm{~s}, 1 \mathrm{H}), 6.92-6.99(\mathrm{~m}, 4 \mathrm{H}), 7.16-7.19(\mathrm{~m}, 2 \mathrm{H}), 7.26(\mathrm{~s}, 1 \mathrm{H}), 7.44-7.47(\mathrm{~m}, 2 \mathrm{H})$. ${ }^{13} \mathrm{C}$ NMR $\left(\mathrm{CDCl}_{3}, 125 \mathrm{MHz}\right) \delta 56.4,60.1,60.9,63.3,102.1,115.6\left(\mathrm{~d}, J_{\mathrm{C}-\mathrm{F}}=22.0 \mathrm{~Hz}\right)$, $124.8\left(\mathrm{~d}, J_{\mathrm{C}-\mathrm{F}}=7.9 \mathrm{~Hz}\right), 126.4,129.3\left(\mathrm{~d}, J_{\mathrm{C}-\mathrm{F}}=8.1 \mathrm{~Hz}\right), 131.1,133.1,133.4,146.1,148.2$, $155.6,160.0\left(\mathrm{~d}, J_{\mathrm{C}-\mathrm{F}}=244 \mathrm{~Hz}\right), 162.4\left(\mathrm{~d}, J_{\mathrm{C}-\mathrm{F}}=246 \mathrm{~Hz}\right), 167.5$. Low resolution mass spectra (EI): $\mathrm{m} / \mathrm{z}: 411(\mathrm{M}+), 380,316,216,109$. HRMS for $\mathrm{C}_{23} \mathrm{H}_{19} \mathrm{O}_{4} \mathrm{NF}_{2}$, calcd: 411.1282, found: 411.1271 .

2-Benzyl-4,5,6-trimethoxy-3-phenyl-2,3-dihydro-isoindol-1-one (15): White solid, mp: $132-134{ }^{\circ} \mathrm{C} .{ }^{1} \mathrm{H}$ NMR $\left(\mathrm{CDCl}_{3}, 500 \mathrm{MHz}\right) \delta 3.23(\mathrm{~s}, 3 \mathrm{H}), 3.66(\mathrm{~d}, 1 \mathrm{H}, J=14.9 \mathrm{~Hz})$, $3.82(\mathrm{~s}, 3 \mathrm{H}), 3.93(\mathrm{~s}, 3 \mathrm{H}), 5.22(\mathrm{~s}, 1 \mathrm{H}), 5.32(\mathrm{~d}, 1 \mathrm{H}, J=14.9 \mathrm{~Hz}), 7.10(\mathrm{~d}, 2 \mathrm{H}, J=6.4$ $\mathrm{Hz}), 7.16(\mathrm{~d}, 2 \mathrm{H}, J=7.1 \mathrm{~Hz}), 7.22-7.35(\mathrm{~m}, 7 \mathrm{H}) .{ }^{13} \mathrm{C} \mathrm{NMR}\left(\mathrm{CDCl}_{3}, 125 \mathrm{MHz}\right) \delta 43.8$, $56.4,60.0,60.8,61.6,102.0,126.9,127.5,128.0,128.4,128.6,128.7,132.0,136.9$, 137.2, 145.6, 148.5, 155.2, 168.1. HRMS for $\mathrm{C}_{24} \mathrm{H}_{23} \mathrm{O}_{4} \mathrm{~N}$, calcd: 389.1627, found: 389.1631 .

5-Methyl-4-phenyl-4,5-dihydro-thieno[2,3-c]pyrrol-6-one (19): Yellow solid, mp: $112-115^{\circ} \mathrm{C} .{ }^{1} \mathrm{H}$ NMR $\left(\mathrm{CDCl}_{3}, 500 \mathrm{MHz}\right) \delta 2.95(\mathrm{~s}, 3 \mathrm{H}), 5.30(\mathrm{~s}, 1 \mathrm{H}), 6.82(\mathrm{~d}, 1 \mathrm{H}, J=4.8$ $\mathrm{Hz})$, 7.15-7.18 (m, 2H), 7.35-7.40 (m, 3H), 7.57-7.59 (m, 1H). ${ }^{13} \mathrm{C}$ NMR $\left(\mathrm{CDCl}_{3}, 125\right.$ MHz) $\delta 28.1,65.3,120.8,127.3,128.8,129.1,129.2,134.9,135.9,155.9,164.8$. Low resolution mass spectra (EI): m/z: $229(\mathrm{M}+), 200,171,152,118,77$. HRMS for $\mathrm{C}_{13} \mathrm{H}_{11} \mathrm{ONS}$, calcd: 229.0561, found: 229.0558 . 
5,6-Dihydro-10,11,12-trimethoxyisoindolo[1,2-a]isoquinolin-8(12bH)-one (21): White solid, mp: $169-174^{\circ} \mathrm{C} .{ }^{1} \mathrm{H}$ NMR $\left(\mathrm{CDCl}_{3}, 500 \mathrm{MHz}\right) \delta$ $\mathrm{m}, 2 \mathrm{H}), 3.65-$ $3.72(\mathrm{~m}, 2 \mathrm{H}), 3.96(\mathrm{~s}, 3 \mathrm{H}), 4.01(\mathrm{~s}, 3 \mathrm{H}), 4.10(\mathrm{~s}, 3 \mathrm{H}), 5.70(\mathrm{~s}, 1 \mathrm{H}), 7.21-7.31(\mathrm{~m}, 5 \mathrm{H})$, 7.71-7.74 (m, 1H). ${ }^{13} \mathrm{C}$ NMR $\left(\mathrm{CDCl}_{3}, 125 \mathrm{MHz}\right) \delta 28.9,39.0,56.4,58.4,60.7,61.2$, $101.8,126.5,126.8,127.6,128.3,128.5,128.8,135.2,135.3,145.0,148.5,155.1,167.7$. Low resolution mass spectrum (EI): m/z: $325(\mathrm{M}+), 310,294,224,139$. HRMS for $\mathrm{C}_{19} \mathrm{H}_{19} \mathrm{O}_{4} \mathrm{~N}$, calcd: 325.1314 , found: 325.1312 .

Compound 23: White solid, mp: $146-149^{\circ} \mathrm{C} .{ }^{1} \mathrm{H}$ NMR $\left(\mathrm{CDCl}_{3}, 500 \mathrm{MHz}\right) \delta 2.98-$ $3.02 \mathrm{~m}, 1 \mathrm{H}), 3.10-3.14(\mathrm{~m}, 1 \mathrm{H}), 3.61-3.65(\mathrm{~m}, 1 \mathrm{H}), 3.99(\mathrm{~s}, 3 \mathrm{H}), 4.22-4.25(\mathrm{~m}, 1 \mathrm{H})$, $5.75(\mathrm{~s}, 1 \mathrm{H}), 7.20-7.37(\mathrm{~m}, 4 \mathrm{H}) 3.96(\mathrm{~s}, 3 \mathrm{H}), 4.01(\mathrm{~s}, 3 \mathrm{H}), 4.10(\mathrm{~s}, 3 \mathrm{H}), 5.70(\mathrm{~s}, 1 \mathrm{H}), 7.26-$ $7.35(\mathrm{~m}, 4 \mathrm{H}), 7.42-7.47(\mathrm{~m}, 1 \mathrm{H}), 7.49(\mathrm{~d}, J=15.1 \mathrm{~Hz}, 1 \mathrm{H}), 7.71(\mathrm{~d}, J=7.5 \mathrm{~Hz}, 1 \mathrm{H}), 7.95$ $(\mathrm{d}, J=7.9 \mathrm{~Hz}, 1 \mathrm{H}) .{ }^{13} \mathrm{C} \mathrm{NMR}\left(\mathrm{CDCl}_{3}, 125 \mathrm{MHz}\right) \delta 29.2,30.1,39.2,56.7,111.1,120.7$, 120.8, 122.0, 124.1, 125.9, 126.8 (two carbons), 127.4, 128.7, 134.7, 135.0, 135.3, 142.6, 162.6. Low resolution mass spectrum (EI): m/z: 288 (M-1), 259, 232, 217.

Compound 25: ${ }^{1} \mathrm{H}$ NMR $\left.\left(\mathrm{CDCl}_{3}, 500 \mathrm{MHz}\right) \delta 3.05-3.11 \mathrm{~m}, 1 \mathrm{H}\right), 3.33-3.39(\mathrm{~m}, 1 \mathrm{H})$, 3.63-3.68 (m, 1H), 4.66-4.71 (m, 1H), $6.12(\mathrm{~s}, 1 \mathrm{H}), 6.83(\mathrm{~d}, J=7.8 \mathrm{~Hz}, 1 \mathrm{H}), 7.06-7.08$ (m, 1H), 7.18-7.25 (m, 2H), 7.55 (d, $J=7.1 \mathrm{~Hz}, 1 \mathrm{H}), 7.58-7.61(\mathrm{~m}, 1 \mathrm{H}), 7.65-7.68(\mathrm{~m}$, $1 \mathrm{H}), 7.93(\mathrm{~d}, J=8.2 \mathrm{~Hz}, 1 \mathrm{H}), 8.01(\mathrm{~d}, J=8.2 \mathrm{~Hz}, 1 \mathrm{H}), 8.34(\mathrm{~d}, J=6.5 \mathrm{~Hz}, 1 \mathrm{H}) .{ }^{13} \mathrm{C}$ NMR $\left(\mathrm{CDCl}_{3}, 125 \mathrm{MHz}\right) \delta 27.4,42.6,59.6,123.8,124.5,124.8,126.1,126.3,126.4$, $126.5,127.1,127.7,128.1,128.2,128.7,131.5,132.8,138.6,143.6,163.2$. Low resolution mass spectrum (EI): m/z: $285(\mathrm{M}+), 284,269,127,77$.

1,4-Dimethyl-5-phenyl-4,5-dihydro-1H-pyrrolo[4,3,2-de]isoquinolin-3-one (27): Yellow solid, mp: $195-197^{\circ} \mathrm{C} .{ }^{1} \mathrm{H}$ NMR $\left(\mathrm{CDCl}_{3}, 500 \mathrm{MHz}\right) \delta 2.84$ (s, 3H), 3.35 (s, 3H), $5.06(\mathrm{~s}, 1 \mathrm{H}), 7.15-7.18(\mathrm{~m}, 2 \mathrm{H}), 7.24-7.38(\mathrm{~m}, 6 \mathrm{H}), 8.06-8.10(\mathrm{~m}, 1 \mathrm{H}) .{ }^{13} \mathrm{C} \mathrm{NMR}\left(\mathrm{CDCl}_{3}\right.$, $125 \mathrm{MHz}) \delta 27.1,30.6,62.0,110.0,120.6,121.4,121.5,122.6,127.9,129.2,129.3$, 134.4, 141.7, 143.6, 155.0, 166.6. Low resolution mass spectra (EI): m/z: 276 (M+), 247, 158, 118, 77. HRMS for $\mathrm{C}_{18} \mathrm{H}_{16} \mathrm{ON}_{2}$, calcd: 276.1263, found: 276.1258 .

2-Methyl-3-phenyl-2,3-dihydro-dibenzo[de,h] isoquinolin-1-one (29): Yellow solid, 72-75 ${ }^{\circ} \mathrm{C} .{ }^{1} \mathrm{H} \mathrm{NMR}\left(\mathrm{CDCl}_{3}, 500 \mathrm{MHz}\right) \delta 3.22(\mathrm{~s}, 3 \mathrm{H}), 5.92(\mathrm{~s}, 1 \mathrm{H}), 7.22-7.27(\mathrm{~m}, 3 \mathrm{H})$, 7.31-7.39 (m, 4H), 7.53-7.56 (m, 1H), 7.68-7.72 (m, 1H), $7.86(\mathrm{~d}, 1 \mathrm{H}, J=8.1 \mathrm{~Hz}), 8.00$ $(\mathrm{d}, 1 \mathrm{H}, J=8.4 \mathrm{~Hz}), 8.56(\mathrm{~s}, 1 \mathrm{H}) .{ }^{13} \mathrm{C} \mathrm{NMR}\left(\mathrm{CDCl}_{3}, 125 \mathrm{MHz}\right) \delta 34.3,67.8,118.2,124.6$, 125.0, 125.7, 126.1, 127.2, 127.8, 128.0, 128.2, 128.9, 129.0, 129.8, 131.8, 132.6, 132.7, 132.8, 143.0, 165.0. Low resolution mass spectra (EI): m/z: $323(\mathrm{M}+), 246,203,176$. HRMS for $\mathrm{C}_{23} \mathrm{H}_{17} \mathrm{ON}$, calcd: 323.1310, found: 323.1309 . 
2-Methyl-3-phenyl-2,3-dihydro-dibenzo[e,g]isoindol-1-one (32): Off-white solid, mp: 142-145 ${ }^{\circ} \mathrm{C} .{ }^{1} \mathrm{H}$ NMR $\left(\mathrm{CDCl}_{3}, 500 \mathrm{MHz}\right) \delta 2.99$ (s, 3H), $5.50(\mathrm{~s}, 1 \mathrm{H}), 7.20-7.22(\mathrm{~m}, 2 \mathrm{H})$, 7.32-7.35 (m, 2H), 7.37-7.41 (m, 2H), 7.56-7.64 (m, 2H), 7.74-7.81 (m, 2H), 8.65-8.69 $(\mathrm{m}, 2 \mathrm{H}), 9.55(\mathrm{dd}, 1 \mathrm{H}, J=15.7 \mathrm{~Hz}, 1.2 \mathrm{~Hz}) .{ }^{13} \mathrm{C} \mathrm{NMR}\left(\mathrm{CDCl}_{3}, 125 \mathrm{MHz}\right) \delta 27.0,65.6$, $122.7,123.5,124.6,125.0,125.3,126.1,126.9$, 127.3, 127.6, 128.1, 128.3, 128.8, 129.2, 130.8, 132.2, 137.1, 144.0, 169.4. Low resolution mass spectra (EI): m/z: 323 (M+), 294, 246, 176, 118, 77. HRMS for $\mathrm{C}_{23} \mathrm{H}_{12} \mathrm{ON}$, calcd: 323.1310 , found: 323.1308 . 
${ }^{1} \mathrm{H}$ and ${ }^{13} \mathrm{C}$ NMR spectra:<smiles>COc1ccc2c(c1)C(c1ccccc1)N(C)C2=O</smiles>
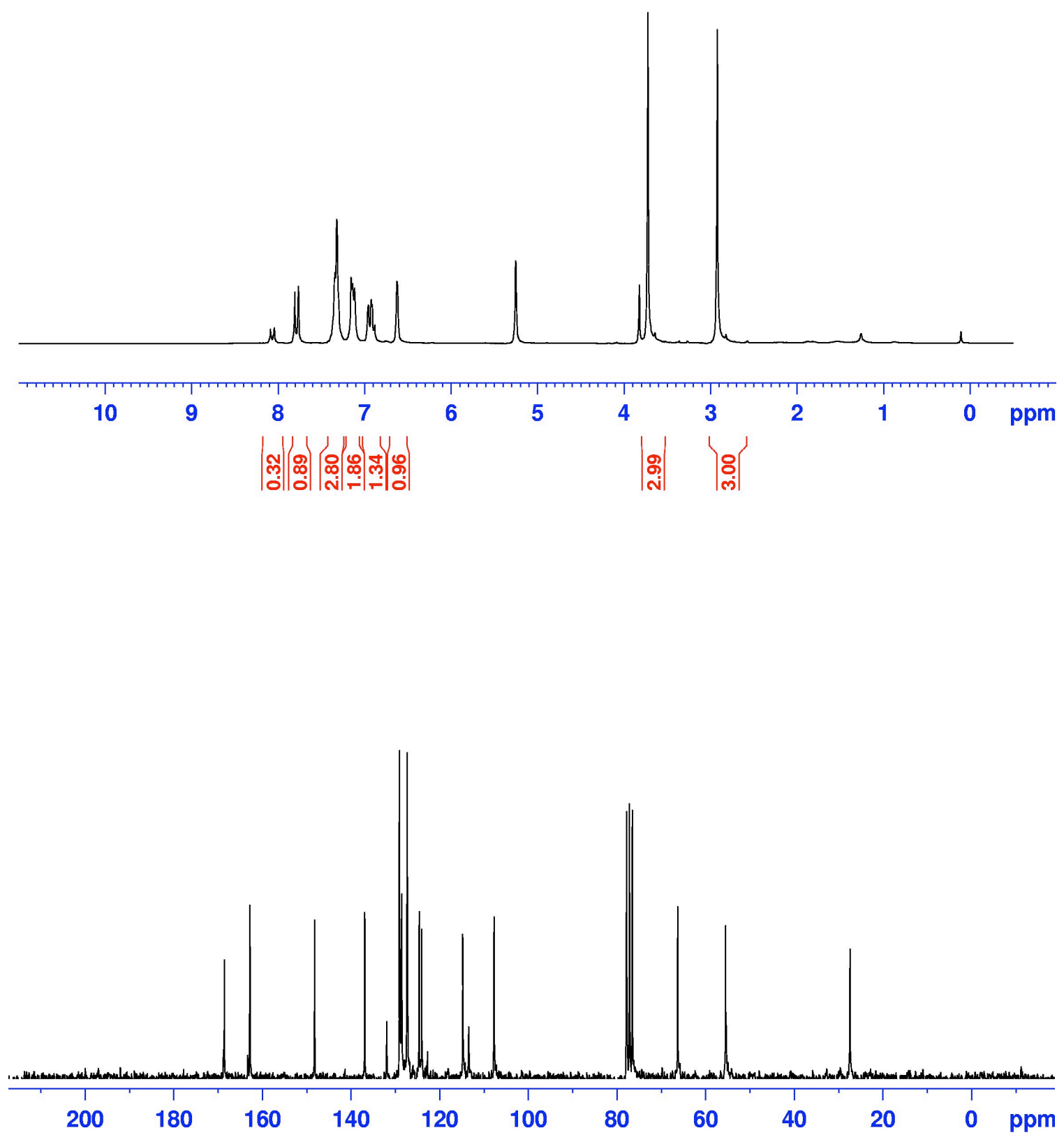

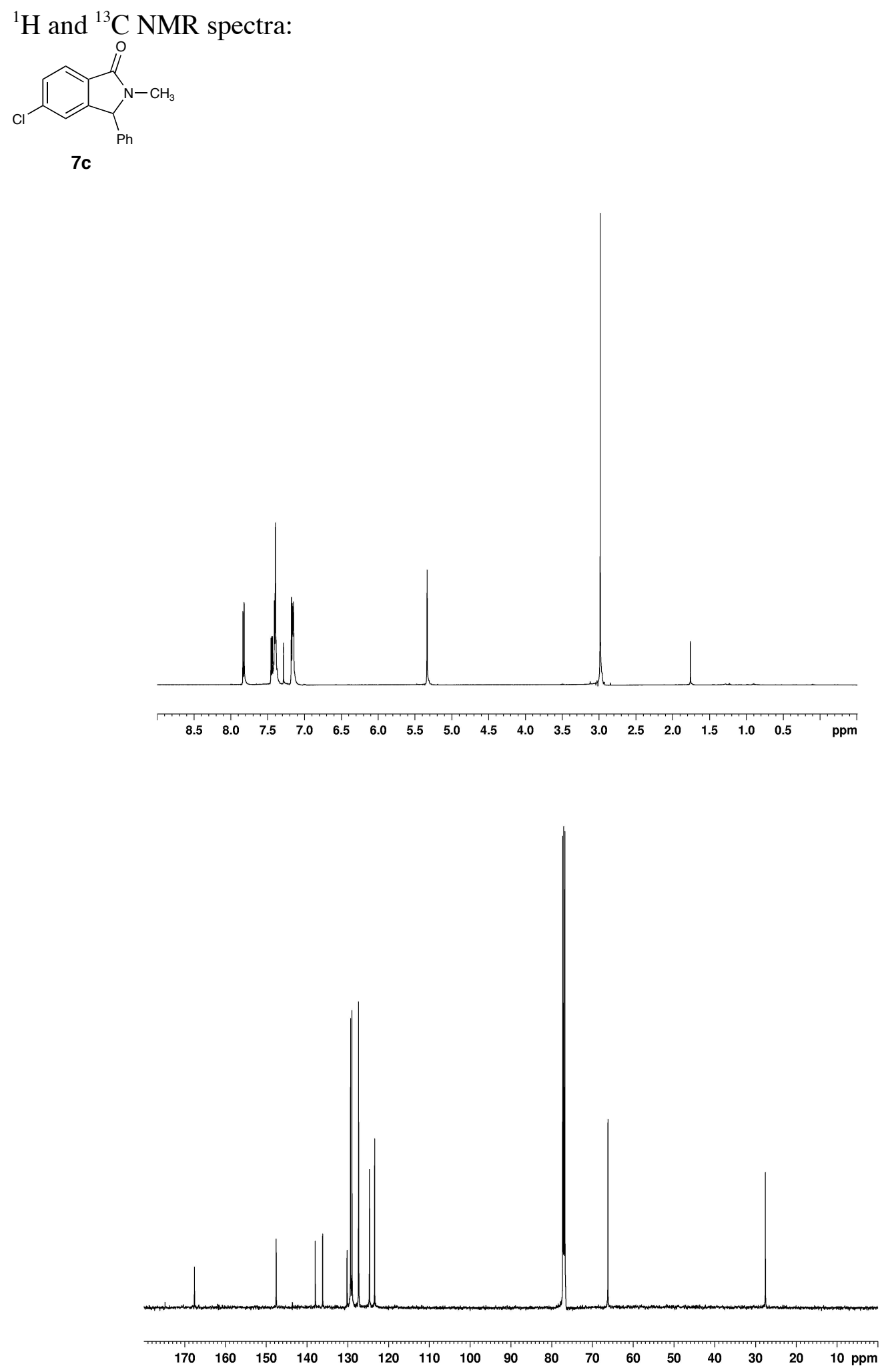

S-7 

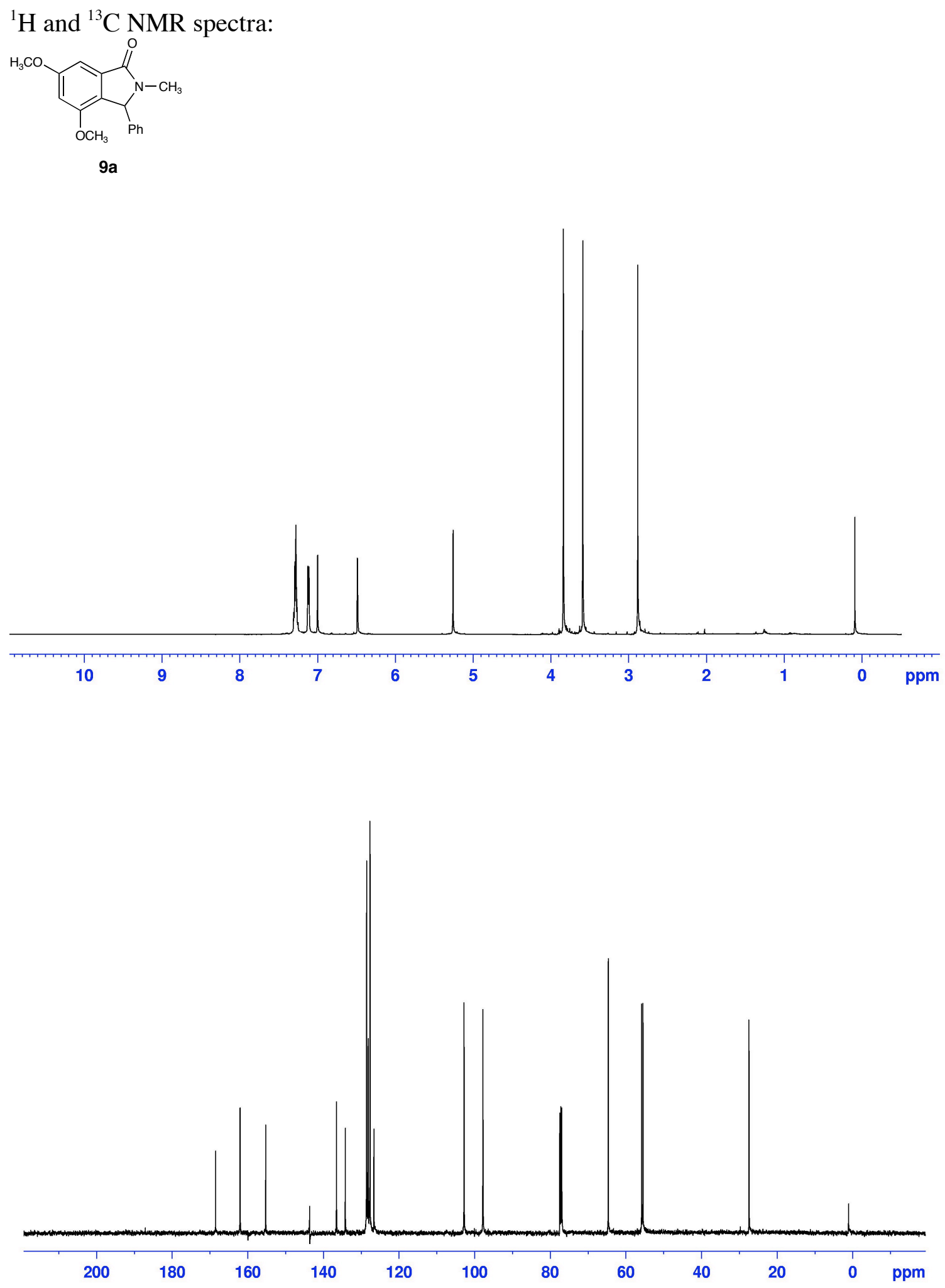

S-8 
${ }^{1} \mathrm{H}$ and ${ }^{13} \mathrm{C}$ NMR spectra:
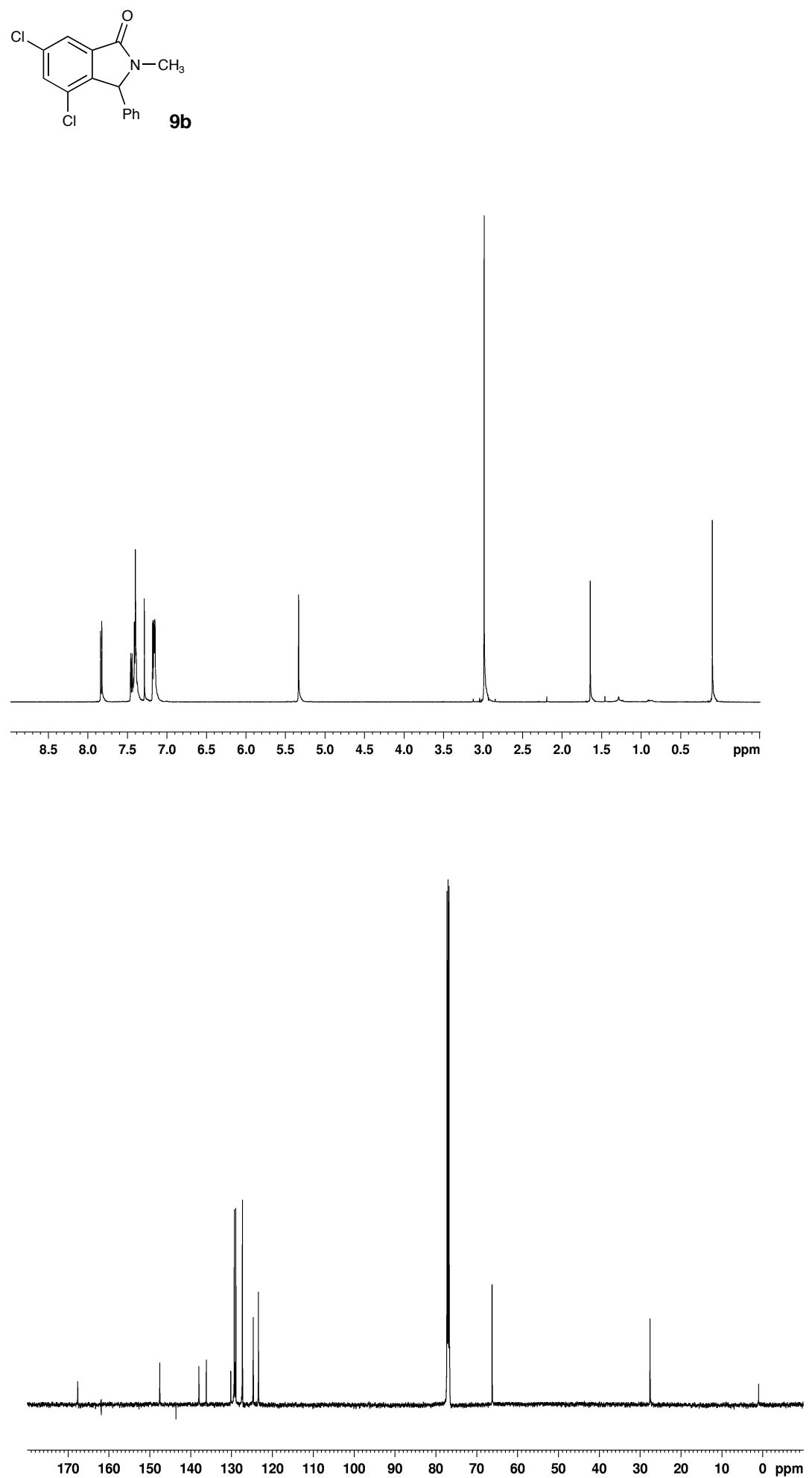

S-9 
${ }^{1} \mathrm{H}$ and ${ }^{13} \mathrm{C}$ NMR spectra:

(N)

11

5-member 3,4,5-trimethoxy-N-Methyl-Phenylamide
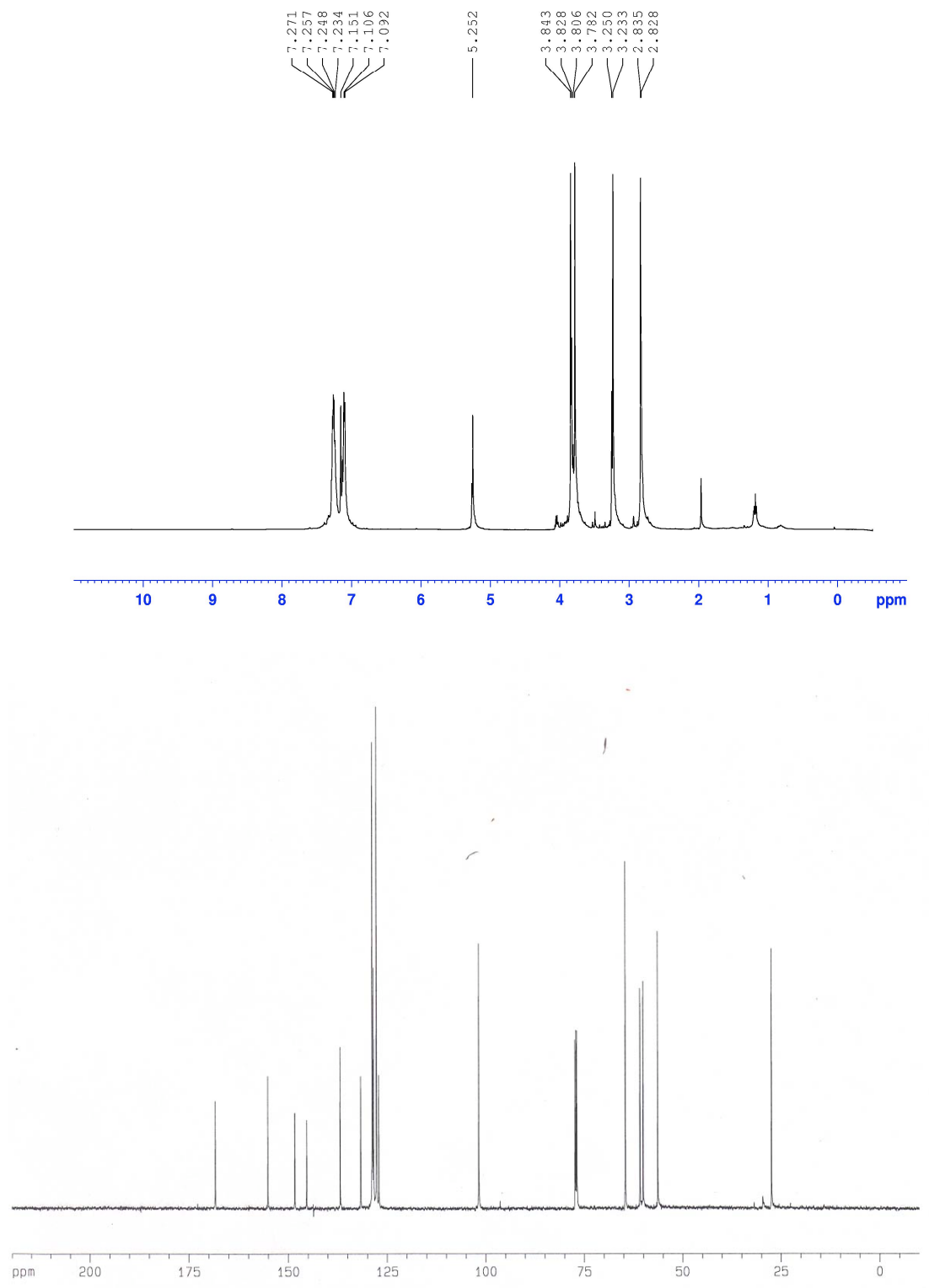

S-10 


\section{${ }^{1} \mathrm{H}$ and ${ }^{13} \mathrm{C}$ NMR spectra:}

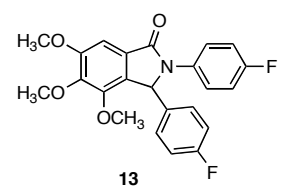

5-member 3, 4,5-trimethoxy-N-p-fluorophenyl-fluorophenylamide
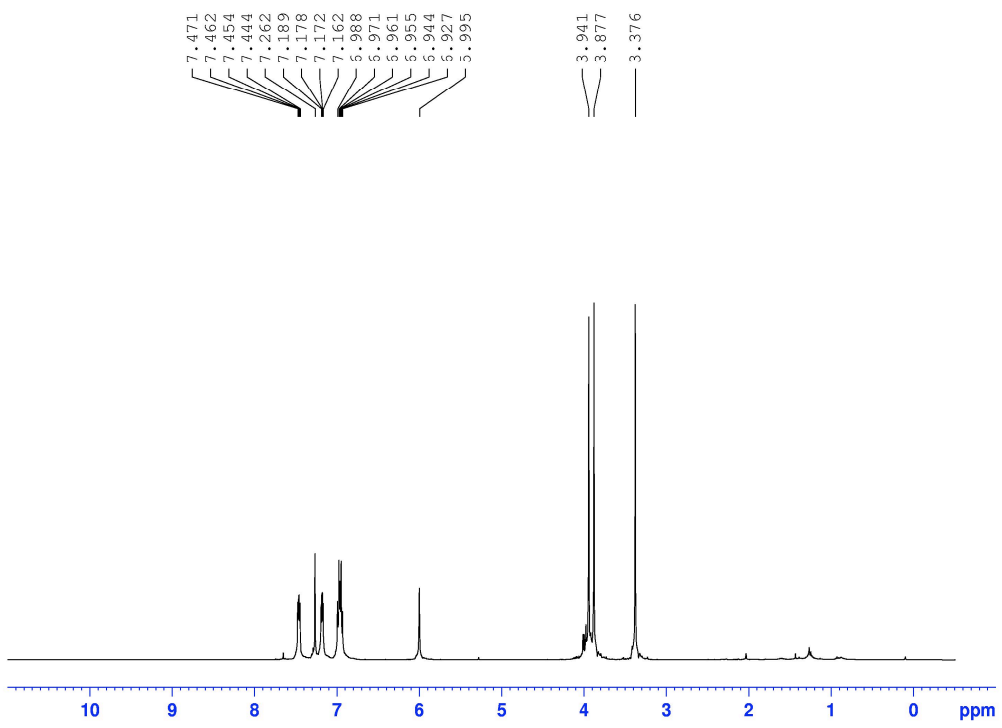

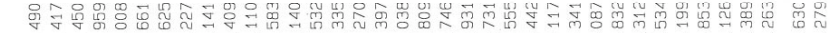

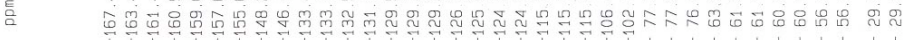
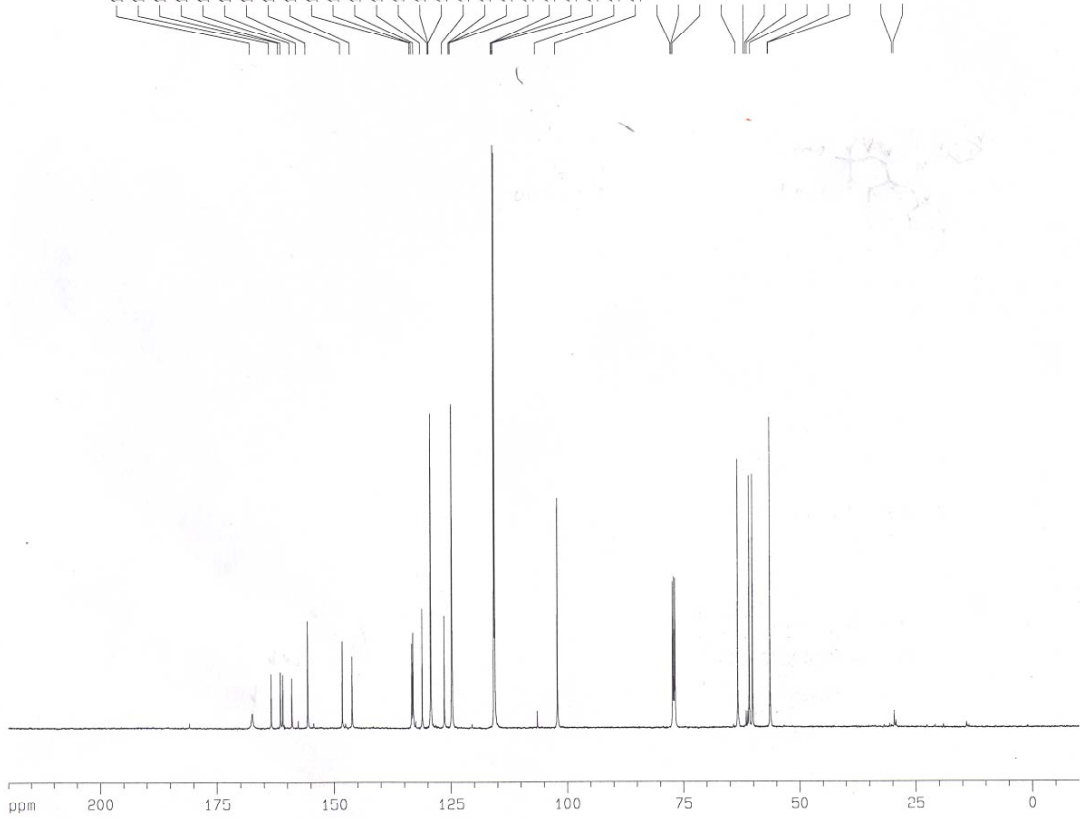

S-11 


\section{${ }^{1} \mathrm{H}$ and ${ }^{13} \mathrm{C}$ NMR spectra:}<smiles>COc1cc2c(c(OC)c1OC)C(c1ccccc1)N(Cc1ccccc1)C2=O</smiles>

15
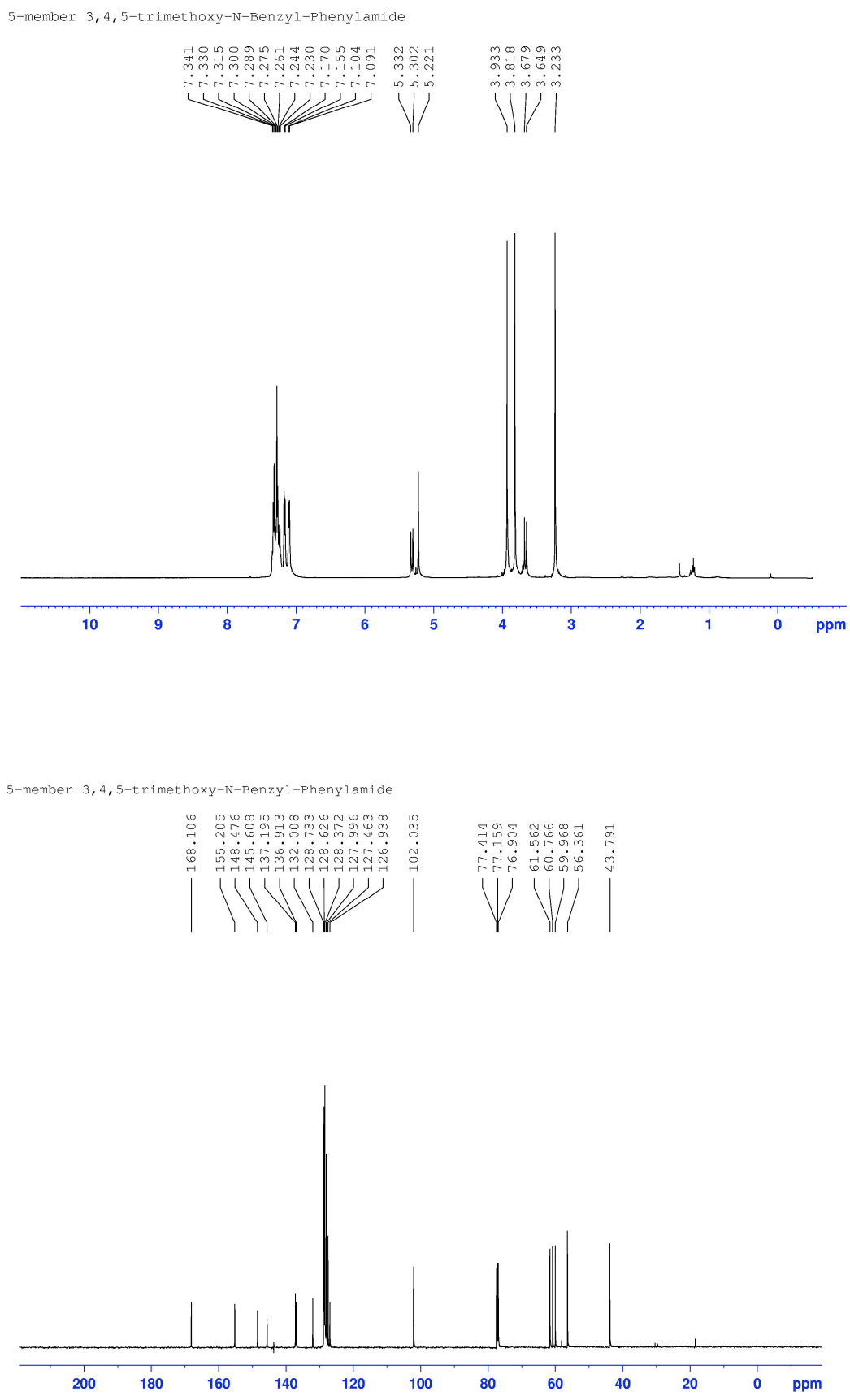

S-12 
${ }^{1} \mathrm{H}$ and ${ }^{13} \mathrm{C}$ NMR spectra:<smiles>CN1C(=O)c2sccc2C1c1ccccc1</smiles>
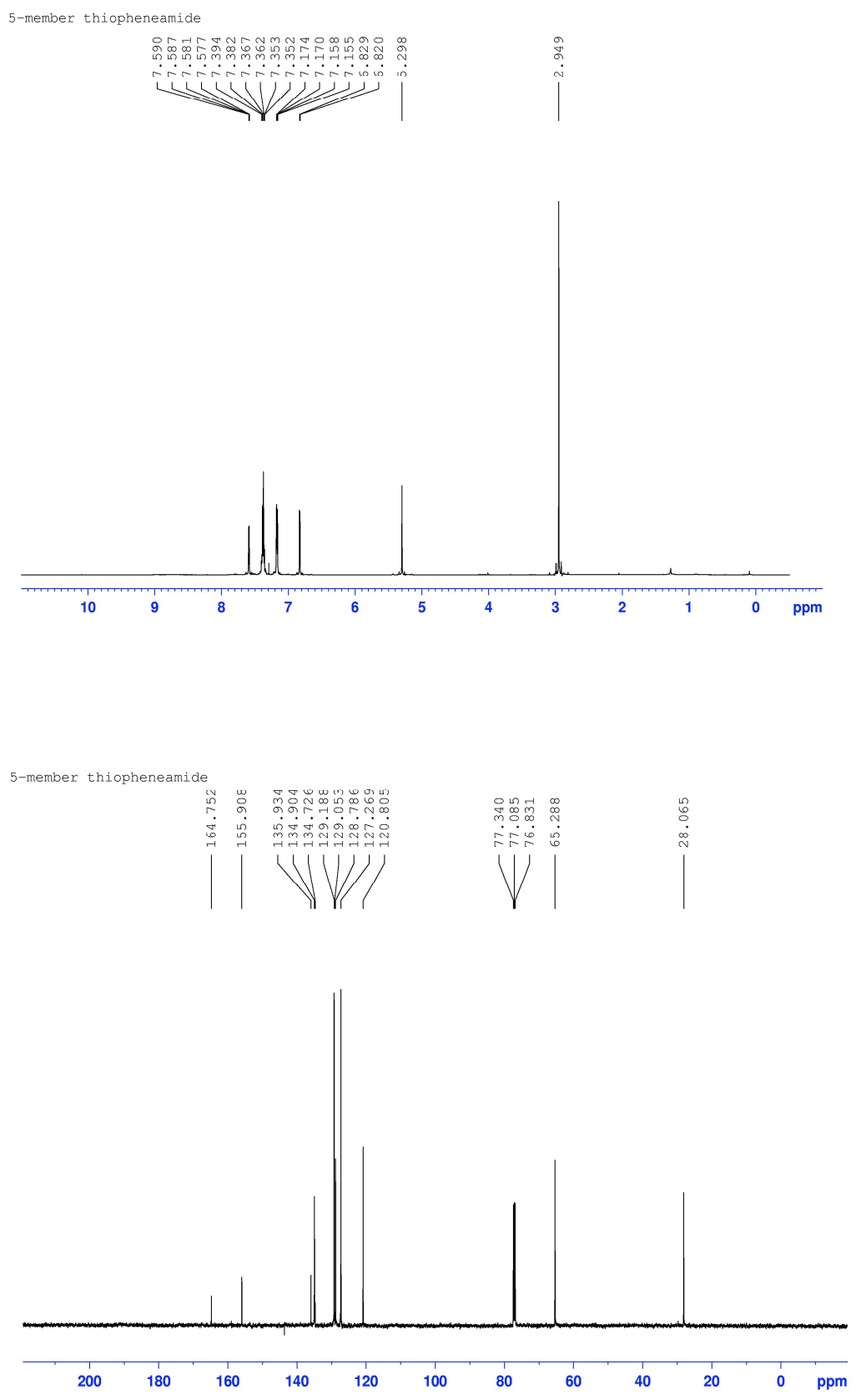

S-13 
${ }^{1} \mathrm{H}$ and ${ }^{13} \mathrm{C}$ NMR spectra:
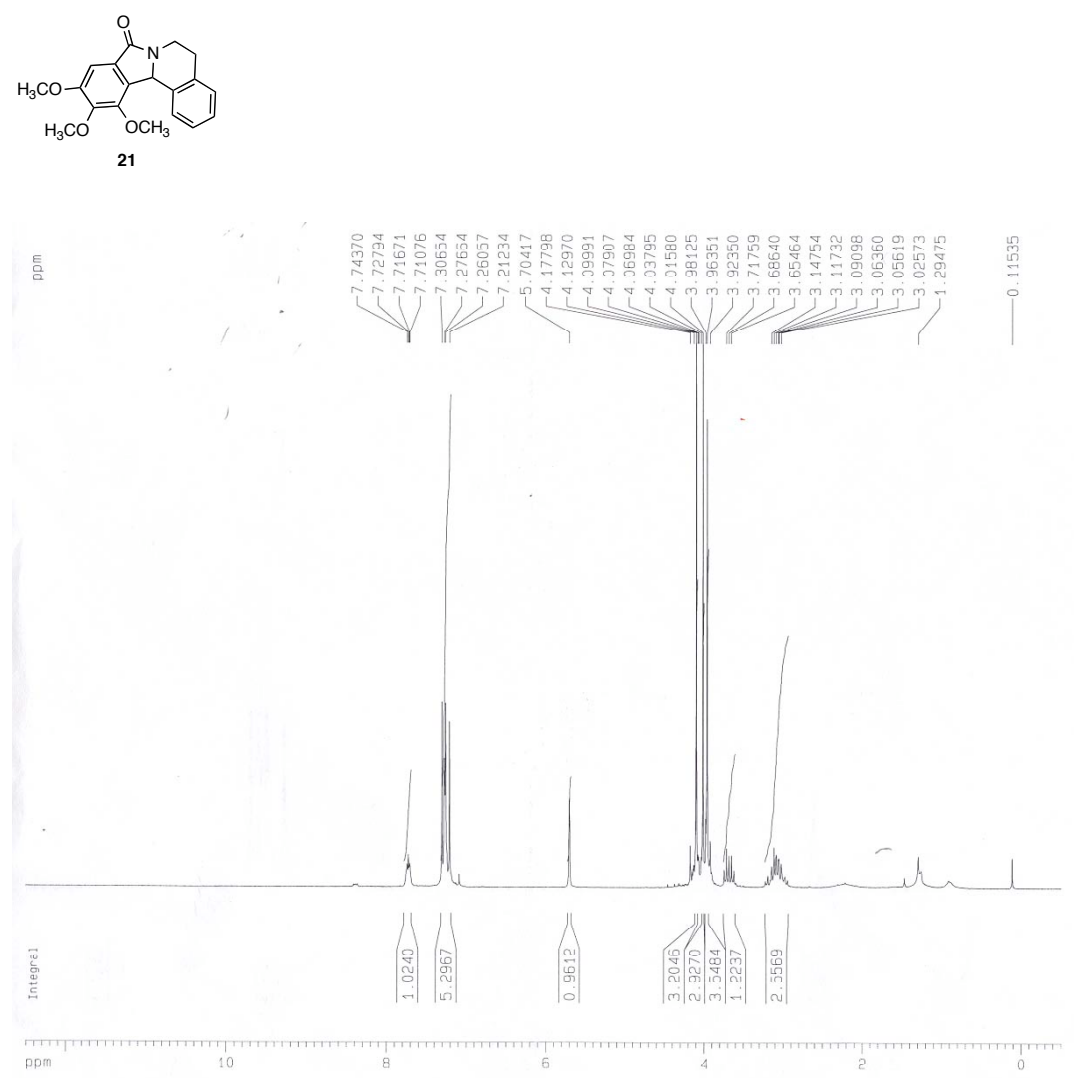

E

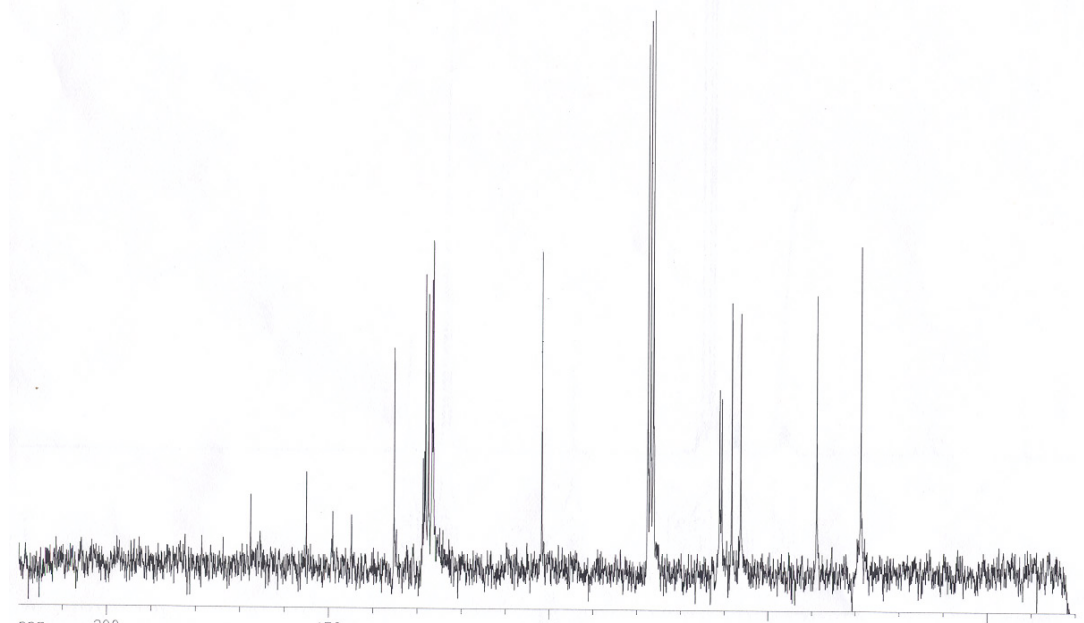

S-14 
${ }^{1} \mathrm{H}$ and ${ }^{13} \mathrm{C}$ NMR spectra:
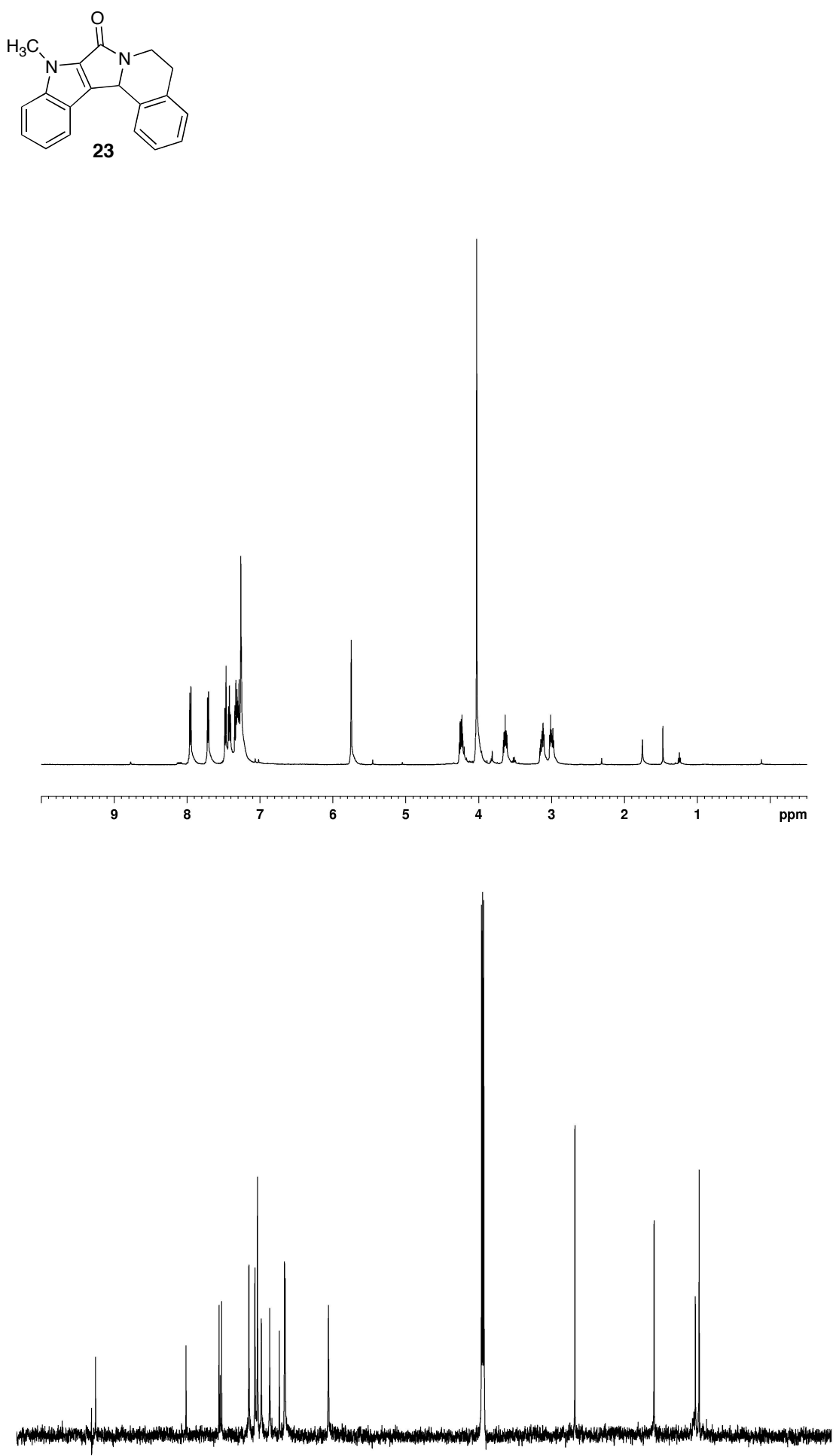

$\begin{array}{lllllllllllllllll}170 & 160 & 150 & 140 & 130 & 120 & 110 & 100 & 90 & 80 & 70 & 60 & 50 & 40 & 30 & 20 & 10 \mathrm{ppm}\end{array}$

S-15 
${ }^{1} \mathrm{H}$ and ${ }^{13} \mathrm{C}$ NMR:
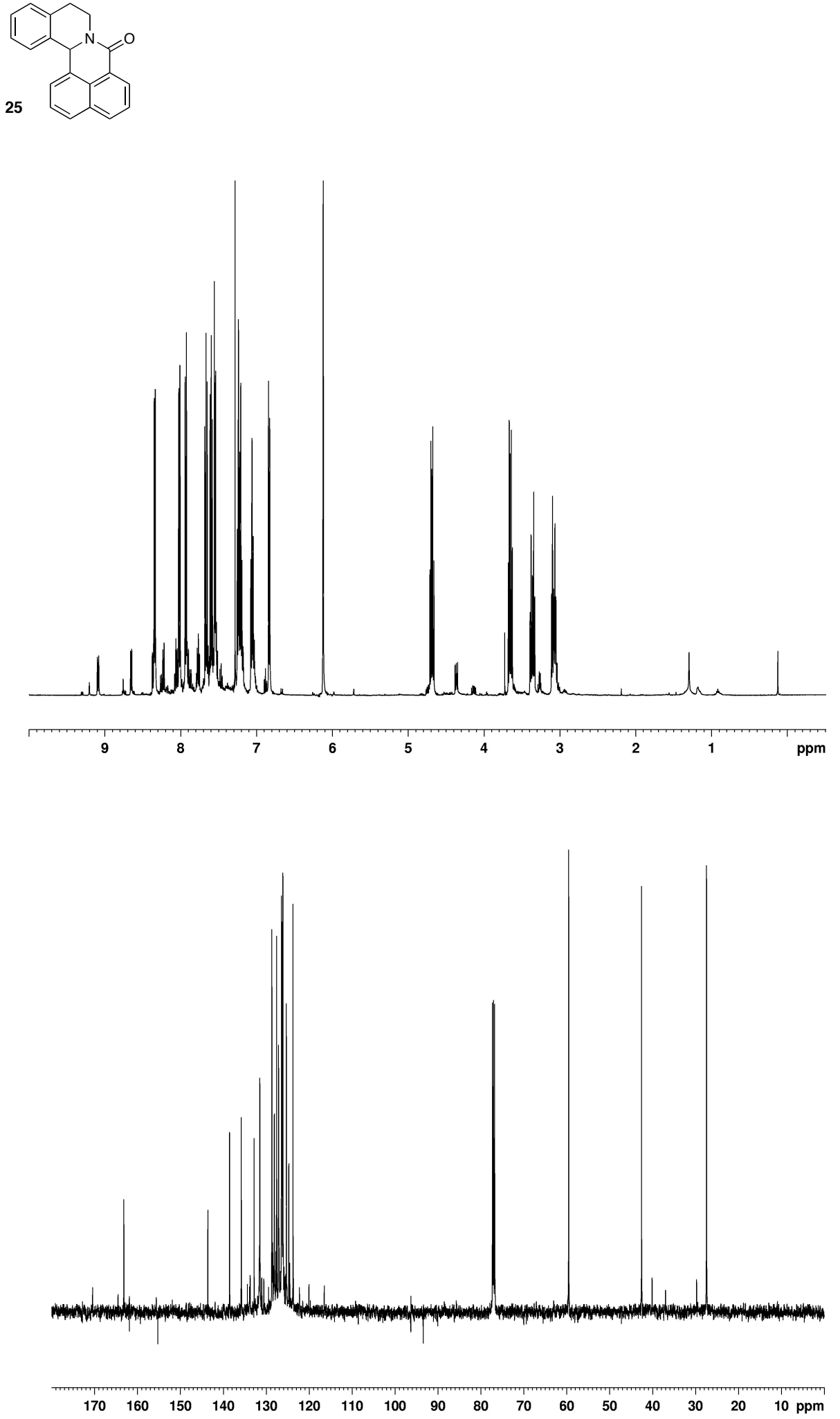

S-16 
COSY NMR:
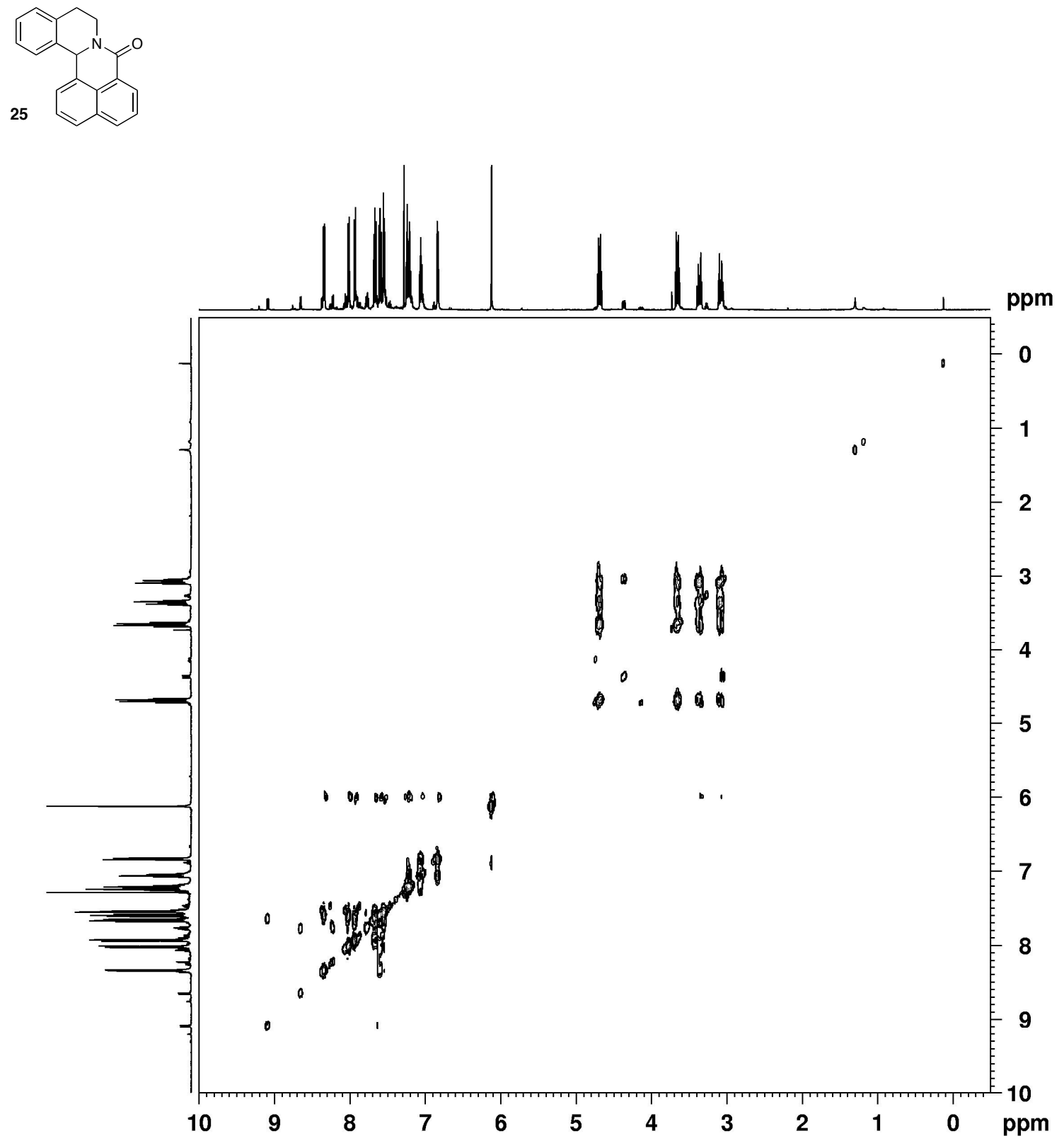

S-17 
${ }^{1} \mathrm{H}$ and ${ }^{13} \mathrm{C}$ NMR spectra:
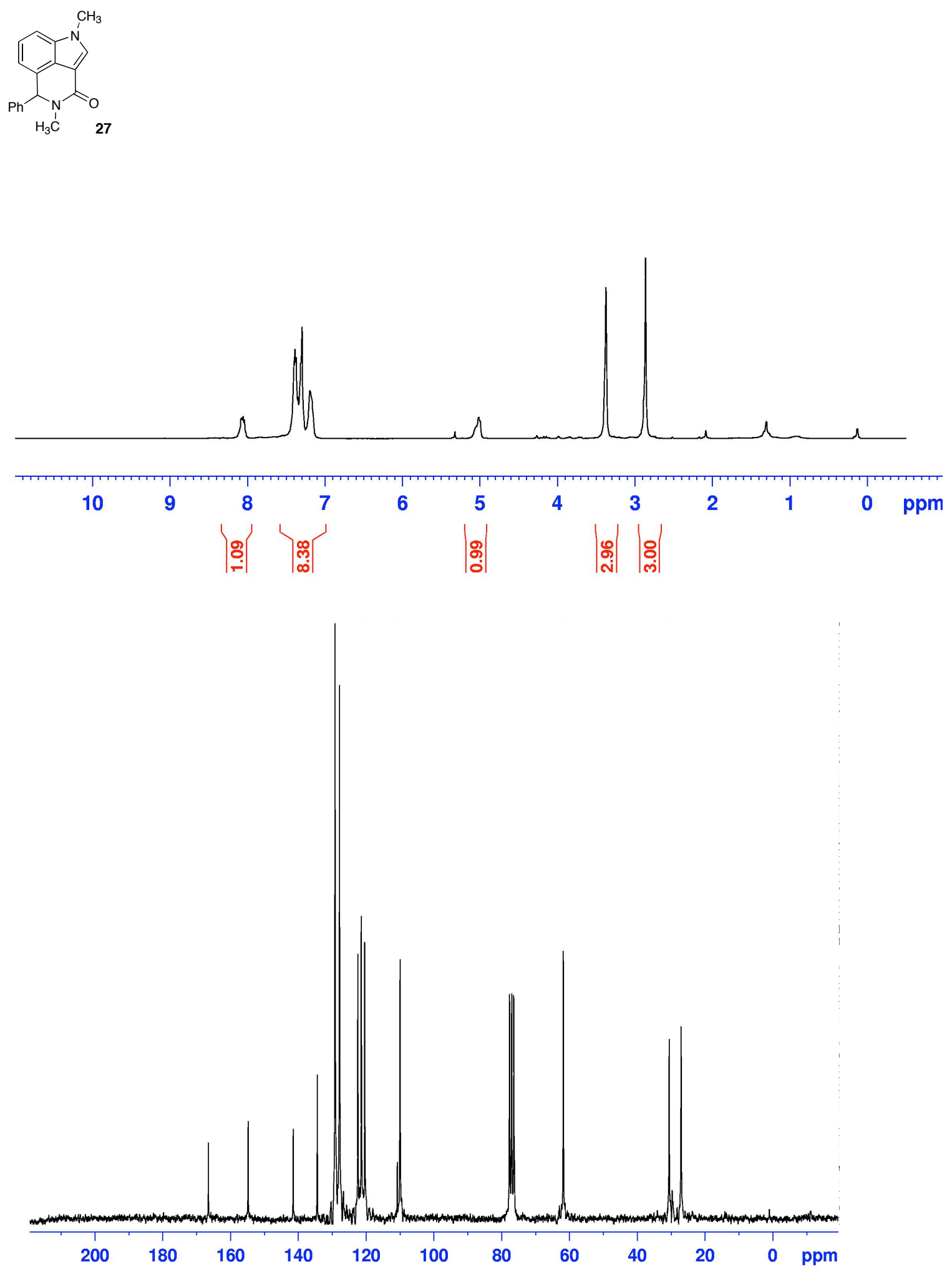

S-18 
${ }^{1} \mathrm{H}$ and ${ }^{13} \mathrm{C}$ NMR spectra:

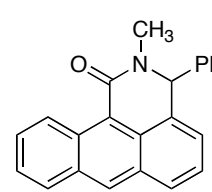

29
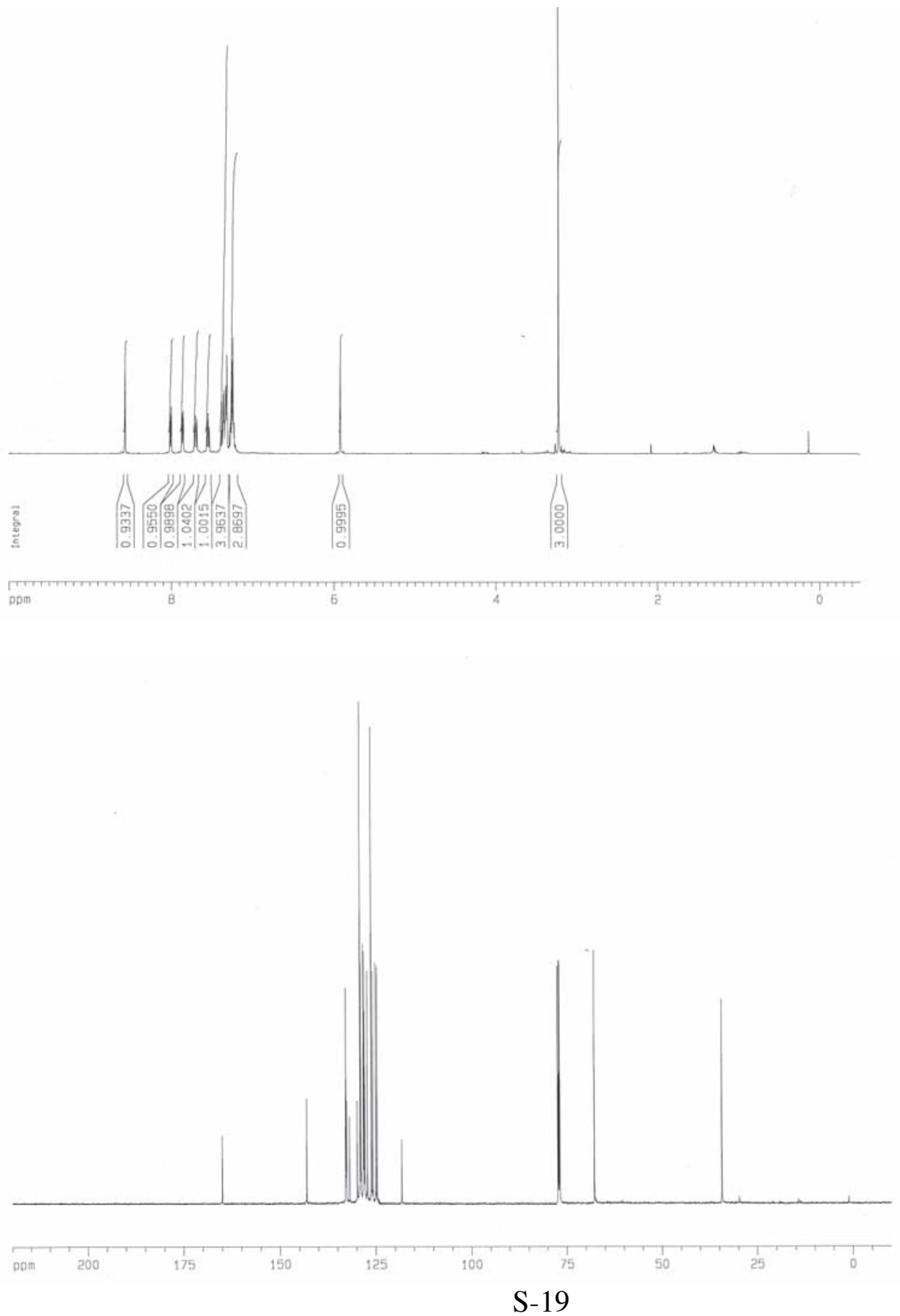
Known compounds and literature citations:

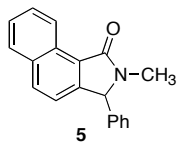

Okura, K.; Inokawa, N.; Miura, M.; Nomura, M. J. Chem. Res., Synop. 1994, 10, $372-$ 373.

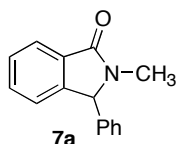

Nan'ya, Seiko; Ishida, Hirofumi; Butsugan, Yasuo. J. Heterocyclic Chem. 1994, 31(6), 1725-1726.

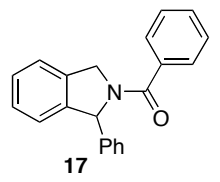

Cohen, Theodore; Dinwoodie, A. H.; McKeever, L. Dennis. J. Org. Chem. 1962, 27 3385-3390.

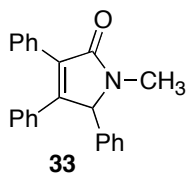

Bouancheau, C.; Rudler, M.; Chelain, E.; Rudler, H.; Vaissermann, J.; Daran, J.-C. J. Organomet. Chem. 1995, 496(1), 127-135. 
Computational protocols: geometry optimizations were performed employing standard techniques at the B3LYP/6-311++G** level using the Gaussian 03 program. 1,2,3 Optimized structures were subsequently checked by vibrational analysis with respect to being true minima on the potential energy surface, as shown by the absence of imaginary frequencies. Located transitions states were found to have a single imaginary frequency (given in Table S1). Energy differences correspond to conditions at $298 \mathrm{~K}$ and $1 \mathrm{~atm}$, with thermal and zero-point corrections.

(1) Calculations done using Gaussian 03, Revision B.04, Frisch, M. J.; Trucks, G. W.; Schlegel, H. B.; Scuseria, G. E.; Robb, M. A.; Cheeseman, J. R.; Montgomery, Jr., J. A.; Vreven, T.; Kudin, K. N.; Burant, J. C.; Millam, J. M.; Iyengar, S. S.; Tomasi, J.; Barone, V.; Mennucci, B.; Cossi, M.; Scalmani, G.; Rega, N.; Petersson, G. A.; Nakatsuji, H.; Hada, M.; Ehara, M.; Toyota, K.; Fukuda, R.; Hasegawa, J.; Ishida, M.; Nakajima, T.; Honda, Y.; Kitao, O.; Nakai, H.; Klene, M.; Li, X.; Knox, J. E.; Hratchian, H. P.; Cross, J. B.; Bakken, V.; Adamo, C.; Jaramillo, J.; Gomperts, R.; Stratmann, R. E.; Yazyev, O.; Austin, A. J.; Cammi, R.; Pomelli, C.; Ochterski, J. W.; Ayala, P. Y.; Morokuma, K.; Voth, G. A.; Salvador, P.; Dannenberg, J. J.; Zakrzewski, V. G.; Dapprich, S.; Daniels, A. D.; Strain, M. C.; Farkas, O.; Malick, D. K.; Rabuck, A. D.; Raghavachari, K.; Foresman, J. B.; Ortiz, J. V.; Cui, Q.; Baboul, A. G.; Clifford, S.; Cioslowski, J.; Stefanov, B. B.; Liu, G.; Liashenko, A.; Piskorz, P.; Komaromi, I.; Martin, R. L.; Fox, D. J.; Keith, T.; Al-Laham, M. A.; Peng, C. Y.; Nanayakkara, A.; Challacombe, M.; Gill, P. M. W.; Johnson, B.; Chen, W.; Wong, M. W.; Gonzalez, C.; and Pople, J. A.; Gaussian, Inc., Wallingford CT, 2004.

(2) (a) Becke, A. D. Phys. Rev. A 1988, 38, 3098. (b) Becke, A. D. J. Chem. Phys. 1993, 98, 5648. (c) Lee, C.; Yang, W.; Parr, R. G. Phys. Rev. B 1988, 37, 785. (d) R. Fletcher Practical Methods of Optimization; Wiley: New York, 1980; vol. 1 .

(3) Raghavachari, K.; Binkley, J. S.; Seegar, R.; Pople, J. A. J. Chem. Phys. 1980, 72,650 . 
Table S1: Thermodynamic parameters for calculated species (B3LYP/6-311++G**).

\begin{tabular}{|c|c|c|c|c|}
\hline Structure & $\begin{array}{c}\text { H(298K) } \\
\text { (hartree) }\end{array}$ & $\begin{array}{c}\text { S(298K) } \\
(\mathbf{c a l} / \mathbf{m o l} \mathbf{k})\end{array}$ & $\begin{array}{c}\text { G(298K) } \\
\text { (hartree) }\end{array}$ & $\begin{array}{c}\text { IMAG } \\
\text { Freq } \\
\left(\mathbf{c m}^{-1}\right)\end{array}$ \\
\hline $\mathbf{4 2 a}$ & -403.595544 & 103.112 & -403.644536 & none \\
\hline TS [42a->43a] & -403.558978 & 96.377 & -403.604769 & $406 \mathrm{i}$ \\
\hline $\mathbf{4 3 a}$ & -403.583474 & 100.024 & -403.630998 & none \\
\hline $\mathbf{4 2 b}$ & -403.743195 & 101.886 & -403.791604 & none \\
\hline TS [42b->43b] & -403.725667 & 97.105 & -403.771805 & $291 \mathrm{i}$ \\
\hline $\mathbf{4 3 b}$ & -403.757842 & 99.174 & -403.804963 & none \\
\hline $\mathbf{4 0 a}$ & -517.943597 & 108.910 & -517.995344 & none \\
\hline TS [40a->41a] & -517.900738 & 100.212 & -517.948352 & $330 \mathrm{i}$ \\
\hline $\mathbf{4 1 a}$ & -517.908793 & 101.016 & -517.956789 & none \\
\hline $\mathbf{4 0 b}$ & -518.112496 & 108.133 & -518.163873 & none \\
\hline TS [40b->41b] & -518.088142 & 100.705 & -518.135990 & $279 \mathrm{i}$ \\
\hline $\mathbf{4 1 b}$ & -518.110400 & 101.813 & -518.158774 & none \\
\hline
\end{tabular}


Figure S-1

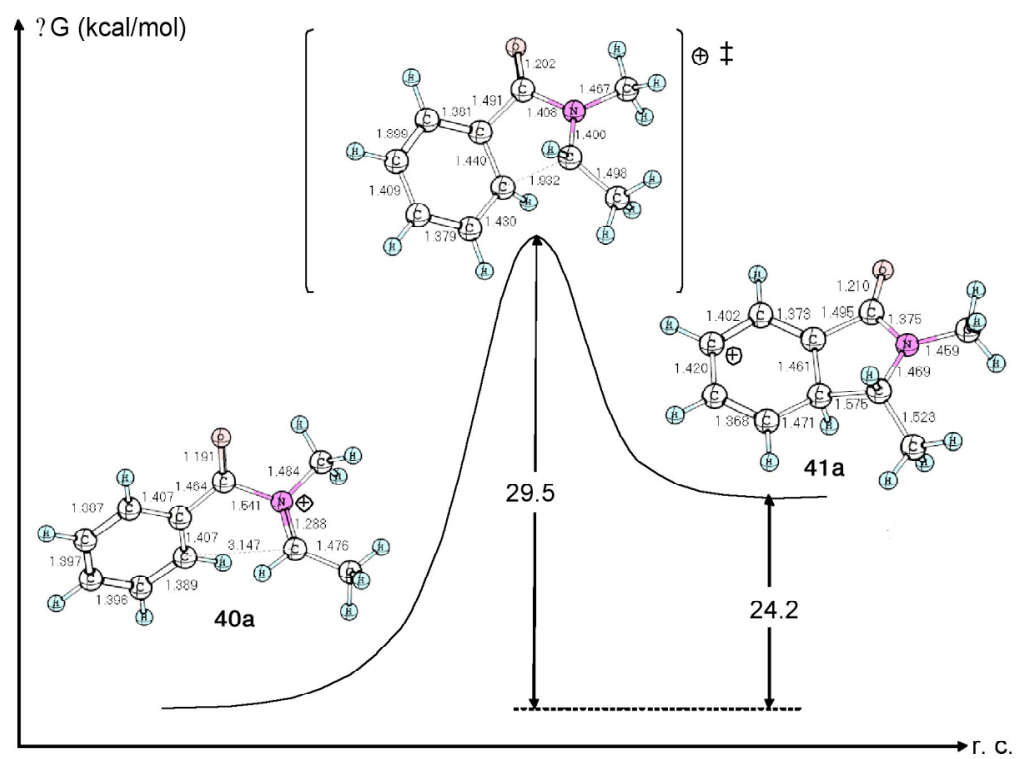

Figure S-2

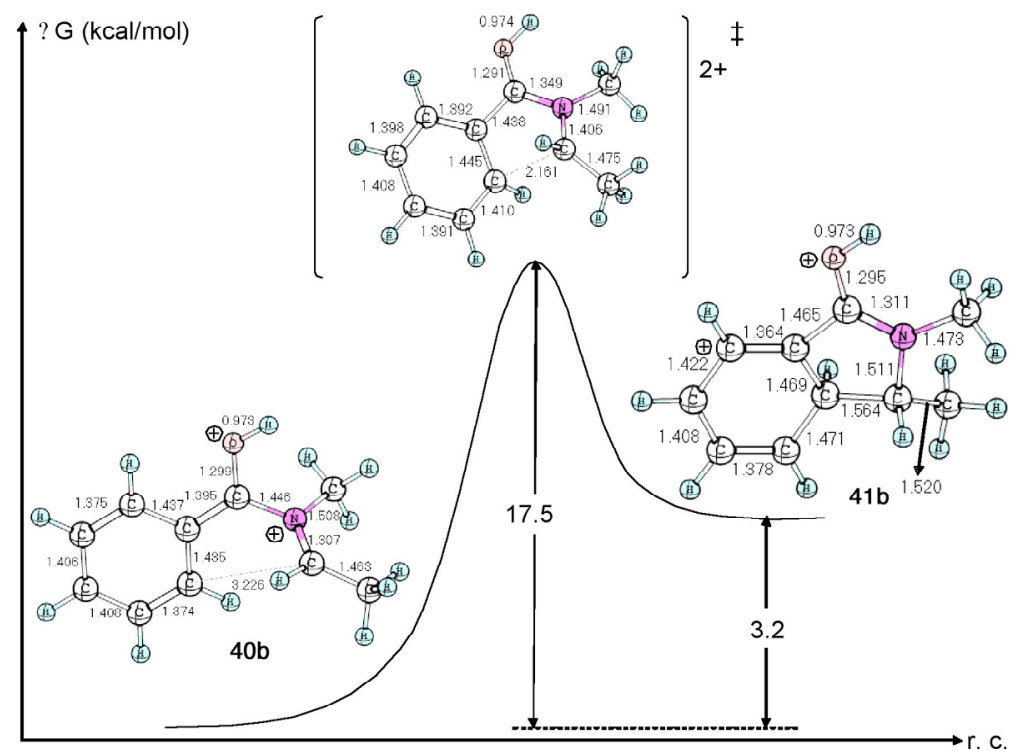


Figure S-3

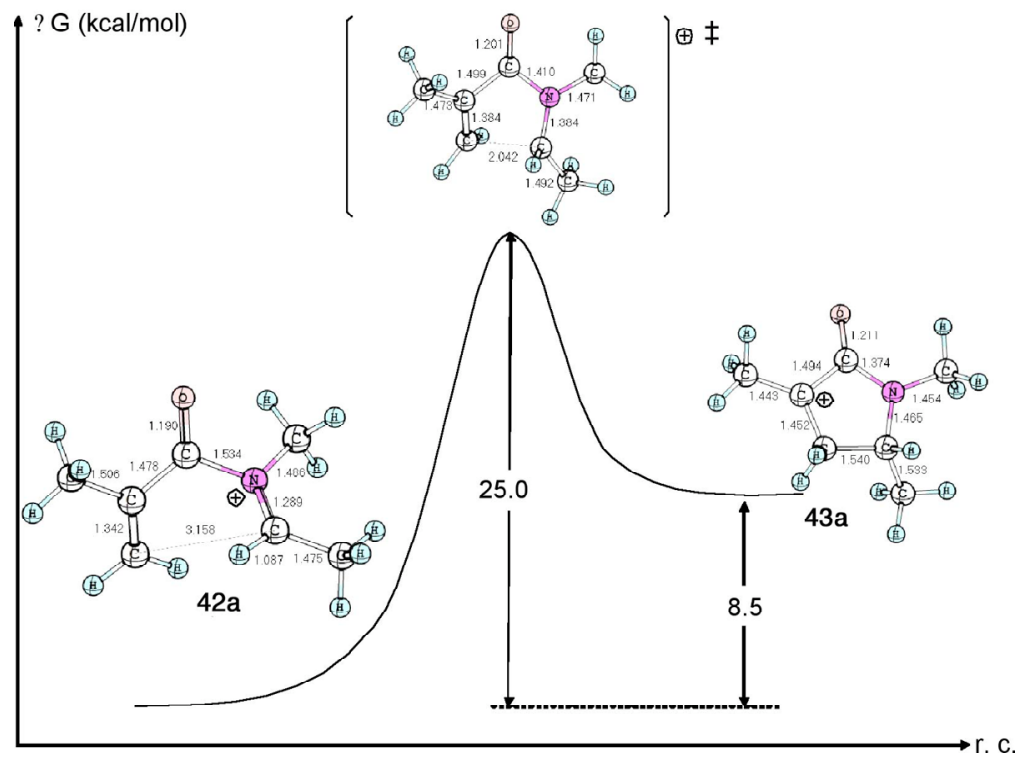

Figure S-4

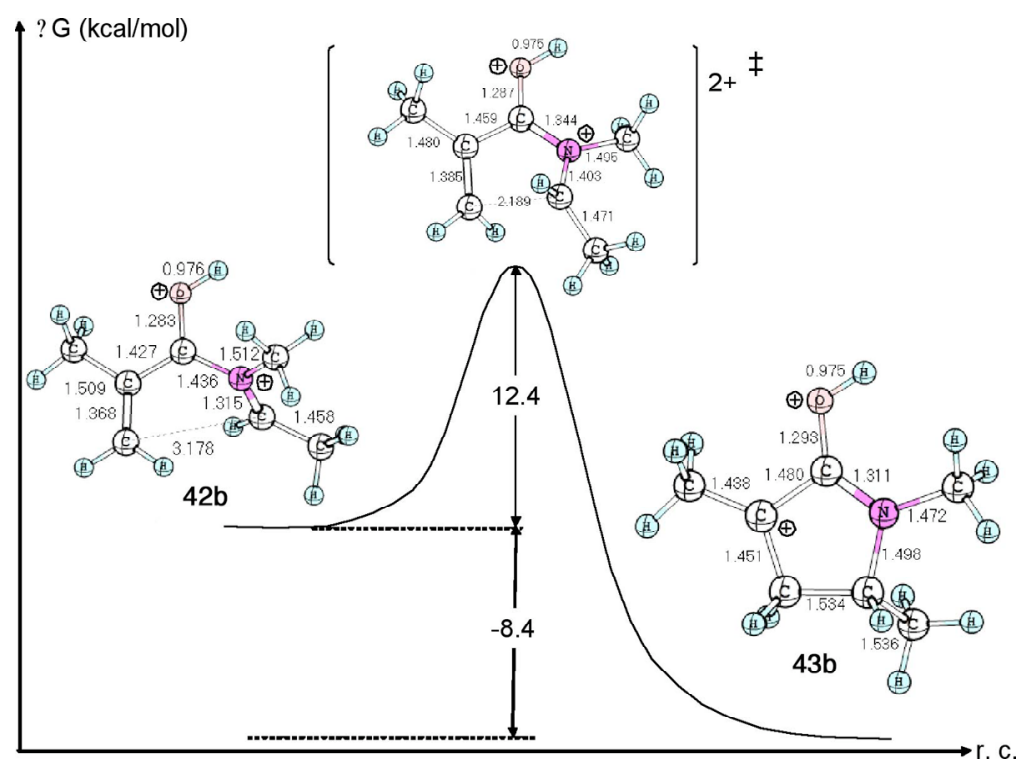

S-24 
Cartesian coordinates:
42a
C,-0.512600082,2.1097133038,2.0395311649
H,-0.6501930562,2.0102932196,3.1155934166
$\mathrm{H}, 0.4219118714,2.6450385659,1.8511538255$
$\mathrm{H},-1.3194956087,2.7263054656,1.635771035$
C, $-0.5040762309,0.7549382037,1.382201625$
C,-0.740794946,-0.397307522,2.028079057
H,- $0.7964897988,-1.3580281663,1.5309669552$
H,-0.9358549006,-0.3992030733,3.0941991961
C,-0.3732522506,0.7891872579,-0.0896373806
O,-0.6086341105,1.7072725312,-0.8088622835
$\mathrm{N}, 0.103109983,-0.4869564915,-0.7959025296$
C,1.1713116452,-1.0842543701,-0.3898511815
H, $1.6020409379,-0.7063782556,0.5344383257$
C,-0.5888713138,-0.8136979203,-2.069229355
$\mathrm{H},-0.9211161339,-1.8507767444,-2.0385776985$
$\mathrm{H},-1.4426998249,-0.150566855,-2.169378051$
$\mathrm{H}, 0.0837847417,-0.6523533862,-2.9109813695$
C,1.8606223675,-2.2009761478,-1.0641289069
$\mathrm{H}, 1.364114886,-2.5678676861,-1.9586278959$
H,2.87686585,-1.872022071,-1.3176496656
H,1.9903989027,-3.019542654,-0.346482236

\section{TS $[42 a->43 a]$}
C, $0.5459138186,2.0826295896,1.8134563822$
$\mathrm{H}, 0.7555878681,1.8939441371,2.8647320948$
H,1.4205770207,2.4969837542,1.3001432932
$\mathrm{H},-0.2194163117,2.8708943615,1.7407814305$
C, $0.0177144324,0.9016031039,1.1096868012$
C,-0.2590218918,-0.336710245,1.6613974099
$\mathrm{H},-1.1310701177,-0.8972909786,1.3390234301$
$\mathrm{H}, 0.0753147995,-0.5682648891,2.669130369$
C,-0.1769923345,0.9638544829,-0.3755186114
O,-0.4723943741,1.9305177831,-1.0236200429
N,0.0134441343,-0.3445554393,-0.8665085816
C, $0.5671379418,-1.2427566606,0.0286315254$
H, $1.5723443287,-1.0230979886,0.3726175472$
C,- $0.5152562693,-0.7106910478,-2.1889280742$
H,-1.5062985438,-1.1634689221,-2.1034919129
$\mathrm{H},-0.5983816191,0.2143003344,-2.7588912846$
$\mathrm{H}, 0.1670930151,-1.3934844095,-2.6917394912$
C, $0.2470074771,-2.6959928949,-0.0738417171$
H,-0.8029690639,-2.8939575657,-0.291093709
$\mathrm{H}, 0.8550671839,-3.1290445475,-0.8789872856$
$\mathrm{H}, 0.5381784469,-3.2113854446,0.8429936368$ 
Cartesian coordinates:
43a
N,-0.8147603339,-0.2389095565,0.426585386
C, $-0.7005898654,1.2038598508,0.1992932579$
C, $0.773683114,1.362499107,-0.218422276$
C, $1.3499971683,0.0298181917,-0.2206391335$
C,0.318925824,-0.9750743292,0.1795485099
C,-1.6999588508,1.7339450309,-0.8349978706
$\mathrm{O}, 0.4899420478,-2.1706674597,0.2623412606$
C, $2.6965209565,-0.3738656216,-0.5461818499$
C,-2.0397995962,-0.873916102,0.8862814891
$\mathrm{H},-0.8731312068,1.7129011473,1.155063012$
H,1.3552385379,2.01463984,0.4544865201
$\mathrm{H}, 0.907901878,1.8301873299,-1.2073426777$
$\mathrm{H}, 2.6343080713,-1.1072625701,-1.3705830562$
H,3.379441599,0.436399665,-0.7889946228
$\mathrm{H}, 3.0768320093,-1.006671124,0.2738040612$
H,-1.7969076061,-1.8787525791,1.22898897
H,-2.4761108156,-0.2898843222,1.6991954057
H,-2.7651012065,-0.9441015357,0.0694336104
$\mathrm{H},-2.7262123321,1.5807726907,-0.4987939857$
$\mathrm{H},-1.5547525323,2.8079847881,-0.9689774364$
H,-1.5703929431,1.2378964781,-1.8004003481

\begin{abstract}
43b
C,1.6027074761,1.6995557649,1.8489016151

$\mathrm{H}, 1.6874907413,1.6211268022,2.9318112985$

$\mathrm{H}, 2.6112494068,1.7238200953,1.4263603075$

$\mathrm{H}, 1.1175228588,2.6465108515,1.6009423944$

C, $0.8233411453,0.5270652349,1.3063687974$

C, $0.4769795011,-0.5595956024,2.0612782843$

H, $-0.000510508,-1.4483989501,1.6638903215$

$\mathrm{H}, 0.7250453651,-0.5860540102,3.1184260881$

C, $0.5500629354,0.498505674,-0.0940923787$

$\mathrm{O}, 1.1733428862,1.3367260383,-0.8391903873$

$\mathrm{N},-0.3617428873,-0.4099394888,-0.7307647793$

C,-1.6131408982,-0.4577835096,-0.3309142579

$\mathrm{H},-1.8734155608,0.2049620457,0.4928939975$

C, $0.1693858298,-1.2183624309,-1.8924542327$

$\mathrm{H},-0.2642884557,-0.8567724953,-2.8256112281$

H,1.2544529097,-1.1315101905,-1.9106499858

H,-0.0929421987,-2.2627834931,-1.7296972746

C,-2.6699848488,-1.2773287334,-0.912546379

H,-2.3777653286,-1.9312475294,-1.7295470511

H,-3.1431552162,-1.8560204956,-0.1027437943

$\mathrm{H},-3.4749702357,-0.5972332708,-1.2398701474$

$\mathrm{H}, 0.9406364996,1.377030371,-1.7865770633$
\end{abstract}


Cartesian coordinates:
TS $[42 b->$ 43b]
C,-2.7391323268,-0.6655542735,0.2968438994
C,-1.3263020196,-0.5741272689,-0.1337669253
C,-0.5449289505,-1.6187902382,-0.599319068
C,- $0.598904566,0.687376434,-0.0367206068$
O,-1.2392294578,1.802317656,-0.0811792983
$\mathrm{N}, 0.7307824524,0.5412632495,0.0958715087$
C, $1.1550190721,-0.7435338263,0.4673202093$
C, $2.4770006673,-1.2738412926,0.0985426492$
C,1.680098617,1.6692172616,-0.1514603881
H,-3.0950728453,-1.6946129501,0.3110731457
$\mathrm{H},-2.9113120273,-0.1785919515,1.2626288046$
H,-3.351084829,-0.1062054125,-0.4312023262
H, $0.1985856431,-1.4680723444,-1.3798095058$
H, $-0.8632185909,-2.6492275992,-0.4602395177$
$\mathrm{H}, 0.6979914074,-1.1346329158,1.3701285963$
H,1.3320391603,2.2563988373,-1.0017729763
H,1.7519170556,2.2769872109,0.7549664319
$\mathrm{H}, 2.6624129724,1.263448958,-0.3784249815$
H,2.8387050487,-0.9700022192,-0.8842829309
H,3.1989926527,-0.9436161609,0.8667972611
$\mathrm{H}, 2.4701952593,-2.3650148773,0.1817649672$
$\mathrm{H},-0.7488953729,2.6212766535,0.1180682381$

\begin{abstract}
43b
C,-0.4251243946,-1.4622787567,0.5366482509

C, $-0.2701396056,-1.0673801315,-0.9374698623$

C, $0.4435265158,0.724410509,0.4009242986$

C, $0.0022090531,-0.3312242916,1.3389199054$

$\mathrm{H},-1.4483447046,-1.7668788898,0.8251916023$

$\mathrm{H}, 0.1683649321,-2.3545385619,0.8216071745$

$\mathrm{H}, 0.4878724588,-1.6837214363,-1.4347104485$

$\mathrm{O}, 0.8779664041,1.8512590692,0.8638503147$

C, $0.0305264257,-0.1833397705,2.7685701028$

$\mathrm{H}, 1.0505586837,0.1160552709,3.0858276182$

$\mathrm{H},-0.5441956975,0.7236962402,3.0496499153$

$\mathrm{H},-0.3159784138,-1.0545253886,3.3205044559$

$\mathrm{N}, 0.3206381508,0.3058569798,-0.8354057831$

C, $0.7002591712,1.079680629,-2.0280989933$

$\mathrm{H}, 1.5823303462,1.6878962763,-1.8165660373$

$\mathrm{H}, 0.9312198922,0.38710264,-2.8362017613$

H,-0.1369243876,1.7198674532,-2.3281096141

C,-1.5673375541,-1.0827852873,-1.759129536

$\mathrm{H},-1.3891532514,-0.7668861327,-2.7875523653$

H,-1.9363222459,-2.1102738988,-1.7951219572

$\mathrm{H},-2.3357323042,-0.4485452312,-1.3104973897$

$\mathrm{H}, 1.134588735,2.5371828444,0.2208317747$
\end{abstract}


Cartesian coordinates:
40a
C,0.335997539,-0.0598267431,1.1020443663
$\mathrm{O}, 0.3939483682,-0.2355961314,2.2788427812$
$\mathrm{N}, 1.7005836043,0.1245815794,0.4100711207$
C, $1.9784465647,-0.5666098781,-0.6411739768$
H,1.1597074901,-1.1555866356,-1.0482821075
C,2.6968898411,0.9374178102,1.1505253035
H,3.0098537287, $1.7749637572,0.5269772796$
$\mathrm{H}, 2.2238182136,1.3012776255,2.0575064261$
$\mathrm{H}, 3.5544251594,0.3235424906,1.4220468661$
C,3.2873967327,-0.6440274475,-1.3194577777
$\mathrm{H}, 4.0491961578,0.0139473066,-0.9096872291$
H,3.6370494483,-1.6835275085,-1.2739057803
H,3.1443778982,-0.4299158402,-2.3846991768
C, $-0.8473174086,-0.0078210933,0.2413009805$
C,-2.0128643173,-0.6252757254,0.7317952734
C,-0.8844687782,0.694808373,-0.977378174
C,-3.1866456415,-0.5651670122,-0.0047876464
$\mathrm{H},-1.9789100803,-1.1460196801,1.6807054086$
C,-2.070583434,0.7648156091,-1.6966590758
H, $-0.0121651094,1.222283921,-1.3444270789$
C,-3.2165284052,0.1284674793,-1.2167582197
$\mathrm{H},-4.080635847,-1.0515616514,0.3655813032$
$\mathrm{H},-2.1064825535,1.3216698287,-2.6248766566$
H,-4.1378428376,0.1809361496,-1.784885669

\section{TS $[40 \mathrm{a}->41 \mathrm{a}]$}

$\mathrm{C}, 0.4225293247,0.1705091994,1.4357887505$

$\mathrm{O}, 0.4675749916,0.3845576407,2.617622384$

$\mathrm{N}, 1.4979290285,-0.2279032922,0.6187891361$

C,1.1392659707,-0.6094450726,-0.6794325174

$\mathrm{H}, 0.5445986958,-1.5173525499,-0.7402152718$

C, $2.8803133317,-0.0801723818,1.0883689696$

H,3.3573217242,0.8004579019,0.6508914507

$\mathrm{H}, 2.8297220151,0.0454102203,2.1693040186$

H,3.4602078503,-0.9720568898,0.8530693433

C, $2.1430740955,-0.4608075667,-1.7807159251$

$\mathrm{H}, 2.627781505,0.5162666526,-1.787031595$

$\mathrm{H}, 2.9183055538,-1.2257670837,-1.6529400325$

$\mathrm{H}, 1.6761928923,-0.6439067172,-2.7489358222$

C,-0.7475228311, $0.3053169475,0.5208417909$

C,-2.0373084788,-0.0570444509,0.8543845942

C,-0.3712871891,0.5845809636,-0.8412970244

C,-2.971429886,-0.2159963446,-0.1751115664

$\mathrm{H},-2.2900211794,-0.2850514453,1.8831437715$

C,-1.3609724604,0.4254156203,-1.8607545579

$\mathrm{H}, 0.3335399874,1.3958920989,-1.0160043186$

C,-2.6296186974,0.0011355563,-1.5247692019

H,-3.9756190412,-0.5456872959,0.0678592059

H,- $1.1262436029,0.6960175139,-2.8838979153$

H,-3.3841486111,-0.1263153097,-2.2915657307 
Cartesian coordinates:
41a
C,-0.3289671032,0.1975988241,-2.2247692795
C,-0.3290482407,0.2477681067,-0.852950048
C,0.899054295,0.2586274394,-0.0616950824
C, $2.1476053126,-0.0282946037,-0.785201746$
C, $2.1304348678,-0.1096212582,-2.1503142645$
C, $0.9080430669,0.0187359767,-2.8605181644$
C,0.4809172102,-0.4127633942,1.2998393446
$\mathrm{N},-0.9711172685,-0.1899893197,1.2879502743$
C,-1.5047101293,0.1455091366,0.0656439265
C,-1.8191385031,-0.4660471799,2.442217152
O,-2.6658495024,0.3036092309,-0.2351340918
C, $1.1848822499,0.1478016532,2.5281097047$
$\mathrm{H}, 0.6847627464,-1.4865804998,1.2114527345$
H,3.0822853961,-0.0741851578,-0.2360056395
H,3.0475424656,-0.2688213441,-2.7040763948
$\mathrm{H}, 0.933257047,-0.0589661249,-3.9432285077$
$\mathrm{H},-1.2581704609,0.2201766348,-2.7822115981$
$\mathrm{H},-2.8546669341,-0.3636127832,2.1210324963$
$\mathrm{H},-1.6248901143,0.243162124,3.2495083698$
H,- $1.6500351503,-1.4830792683,2.8052571684$
$\mathrm{H}, 1.0379282081,1.3320812483,0.2145330983$
$\mathrm{H}, 0.8798938755,-0.3873079285,3.4286055862$
$\mathrm{H}, 0.9659851852,1.2100011852,2.6647241693$
$\mathrm{H}, 2.2662864778,0.022295101,2.4336600743$

\begin{abstract}
40b
C,-1.8005605361,0.0129630385,-2.7154924195

C, $-1.8183106962,-0.0080137361,-1.3406432354$

C,-0.5840683492,0.0946642061,-0.6121498893

C, $0.6512988511,0.2393571145,-1.3287579049$

C,0.6419333716,0.263393654,-2.7027960399

C, $-0.577750056,0.1467147296,-3.3973631$

$\mathrm{H},-2.7248178861,-0.0760865302,-3.2733106128$

H,-2.7517459619,-0.1202221317,-0.8037613062

$\mathrm{H}, 1.5880248657,0.3763275609,-0.8028807422$

$\mathrm{H}, 1.5658637169,0.3886396268,-3.2538251761$

$\mathrm{H},-0.5733036111,0.1673952961,-4.4823205178$

C,-0.63467628,0.0994227452,0.7818882498

O, $-1.7705857364,0.2038682548,1.4030561888$

$\mathrm{N}, 0.5457490757,0.0379280532,1.6140266899$

C,1.3140961362,-1.0187993826,1.5666164487

H,1.0064353003,-1.7964098544,0.8690026789

C, $2.5105258139,-1.2487439052,2.375841264$

$\mathrm{H}, 2.7889258334,-0.4413205021,3.047607515$

H,2.3556917517,-2.1780583069,2.9470746138

H,3.3446742337,-1.4915619083, 1.700465692

C, $0.7906827359,1.2095941582,2.5313237519$

$\mathrm{H}, 0.6919593049,0.8935790857,3.5700946041$

$\mathrm{H}, 1.7922800283,1.5936806571,2.3420199811$

$\mathrm{H}, 0.0643909683,1.9866981716,2.301626677$

H,-1.7629621304,0.0575806922,2.3647675015
\end{abstract}




\begin{abstract}
TS $[40 \mathrm{~b}->$ 41b]
C, $-2.0827030081,-0.0274647716,-2.2239717433$

C, $-2.036988558,0.1885808105,-0.8432908466$

C, $-0.792808554,0.377610517,-0.2477052336$

C, $0.4167480216,0.4277303067,-1.0360941745$

C, $0.3361909706,0.1677188506,-2.4192633288$

C,-0.9067513809,-0.0648038193,-2.998076034

$\mathrm{H},-3.0401997563,-0.2042819378,-2.7019492178$

$\mathrm{H},-2.9383648486,0.1346645328,-0.2436694872$

H,1.2382477156,1.0621329853,-0.7084293014

$\mathrm{H}, 1.2224286463,0.2427464527,-3.0391914819$

$\mathrm{H},-0.9803952662,-0.2320840269,-4.0670115094$

C,-0.5639691306,0.3225229261,1.1705615248

O,-1.4648034162,0.7132790034,2.009113741

$\mathrm{N}, 0.6604936842,-0.1404697691,1.4970825317$

C, $1.3006834757,-0.8504693398,0.4659651742$

$\mathrm{H}, 0.7154718003,-1.663036195,0.0514564799$

C,2.7691635174,-0.9735491148,0.4051823589

H,3.3111514475,-0.0577033869,0.6445542779

$\mathrm{H}, 3.0733578276,-1.7560562831,1.1212383894$

H,3.0702185162,-1.3457964221,-0.5760660318

C, $1.2902313112,0.1098733731,2.8255662364$

H,1.0791188811,-0.7271744407,3.4947792288

$\mathrm{H}, 2.3660960002,0.2027222398,2.6971912942$

$\mathrm{H}, 0.9118703613,1.0473350729,3.2328910526$

$\mathrm{H},-1.3128097745,0.5070893362,2.9484750568$
\end{abstract}
41b
$\mathrm{N},-1.3469449788,-0.0421714073,-0.8867058179$
C,-1.346151159,0.0270202549,0.4226680806
C, $0.0277933289,0.0641933775,0.9291631665$
C,0.9040298439,-0.2967419087,-0.1938634395
C, $0.0442787292,-0.051575209,-1.4765520844$
C, $2.323801985,0.0559880349,-0.041408887$
C, $2.7896477557,0.4788535168,1.1851168989$
C, $1.8997964275,0.6611993467,2.2615828817$
C, $0.495021857,0.4758599103,2.1422368908$
O,-2.345244688,0.0953698839,1.2439627057
$\mathrm{H}, 0.2267083236,0.9647644964,-1.838721701$
C,-2.5394906236,-0.0566354613,-1.7517004939
H,3.0109927198,-0.1324669204,-0.8612650607
$\mathrm{H}, 3.8459550363,0.6758452096,1.3289671813$
$\mathrm{H}, 2.2984164581,0.9905864858,3.2172688689$
$\mathrm{H},-0.1645154326,0.7132572483,2.9695227901$
$\mathrm{H},-3.4030541538,0.3394012634,-1.2164624608$
$\mathrm{H},-2.7479732174,-1.0757995093,-2.0833708598$
$\mathrm{H},-2.3549389672,0.5823545418,-2.615113633$
H,0.9963099098,-1.4137778975,-0.1265310233
C, $0.2157488593,-1.0668452803,-2.5949299513$
$\mathrm{H},-0.3941441048,-0.812677527,-3.4627876154$
$\mathrm{H}, 1.255008091,-1.0546629834,-2.9327320641$
H,-0.029469924,-2.0808678277,-2.2694470103
H,-3.2355844053,0.08838471, 0.8520332929 